\title{
Special Sediment
}

Investigations

Mississippi River at

St.Louis, Missouri, 1961-63

GEOLOGICAL SURVEY WATER-SUPPLY PAPER 1819-J

Prepared in cooperation with the U.S. Army Corps of Engineers, St. Lou is District

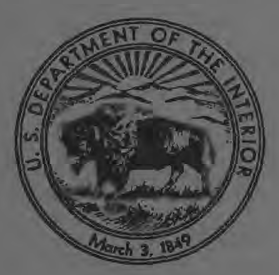




\section{Special Sediment}

Investigations

\section{Mississippi River at}

\section{St.Louis, Missouri, 1961-63}

By CLOYD H. SCOTT and HOWARD D. STEPHENS

CONTRIBUTIONS TO THE HYDROLOGY OF THE UNITED STATES

GEOLOGICAL SURVEY WATER-SUPPLY PAPER 1819-J

Prepared in cooperation with the U.S. Army Corps of Engineers, St. Louis District

Four sets of comprehensive hydraulic

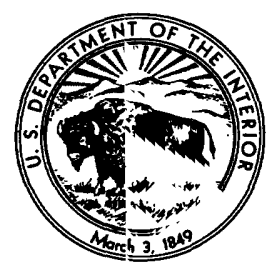
and sediment data are presented and briefly analyzed 
UNITED STATES DEPARTMENT OF THE INTERIOR

STEWART L. UDALL, Secretary

GEOLOGICAL SURVEY

William T. Pecora, Director 


\section{CONTENTS}

$\begin{array}{lr}\text { Abstract } & \text { J1 } \\ \text { Introduction } & \mathbf{1} \\ \text { Hydraulic data } & 2 \\ \text { Streamflow measurements and cross-section areas } & \mathbf{2} \\ \text { Water-surface slopes. } & 7 \\ \text { Energy gradients } & 10 \\ \text { Longitudinal bed profiles } & 10 \\ \text { Vertical distribution of velocity } & 23 \\ \text { Computation of average turbulence constant } & 25 \\ \text { Sediment data } & \mathbf{2 5} \\ \text { Vertical distribution of suspended sediment } & 33 \\ \text { Cross-section suspended-sediment samples } & 34 \\ \text { Bed-material samples } & 35 \\ \text { Summary } & \\ \text { Literature cited } & \end{array}$

\section{ILLUSTRATIONS}

Plate 1-3. Graphs showing-

[Plates are in pocket]

1. Vertical distribution of velocity.

2. Vertical distribution of suspended sediment.

3. Relation of $z_{1}$ to fall velocity.

Figure 1. Map showing study reach of Mississippi River

2. Graph of transverse profiles of riverbed

3. Graphs of longitudinal water-surface and energy profiles

4. Longitudinal profiles of riverbed through quarter points of river width, Apr. 19, 1961, and Apr. 23, 1962

5. Longitudinal profiles of riverbed through quarter points of river width, Oct. 11, 1962, and Apr. 18, 1963

\section{TABLES}

TABLE 1. Streamflow measurements from MacArthur Bridge-..-...

2. Cross-section data obtained with a depth sounder

3. Measured velocity at selected points above riverbed

4. Summary of turbulence constants

5. Summary of energy gradients

6-8. Size distributions of :

6. Suspended stdiment and velocity at selectec points above riverbed

7. Suspended sediment in cross-section samples.......

8. Bed material 


\title{
CONTRIBUTIONS TO THE HYDROLOGY OF THE UNITED STATES
}

\author{
SPECIAL SEDIMENT INVESTIGATIONS \\ MISSISSIPPI RIVER AT \\ ST. LOUIS, MISSOURI, 1961-63
}

By Cloyd H. SCOTT and Howard D. STEPhens

\begin{abstract}
Four sets of comprehensive hydraulic and sediment data were ohtained during 1961-63 for the Mississippi River at St. Louis at ranges of mean velocity from 3.3 to 5.6 feet per second, of mean depth from 22 to $: 7$ feet, of width from 1,570 to 1,670 feet, of mean water-surface slope from 0.000054 to 0.000109 , and of suspended-sediment concentration from 314 to 928 parts per million. The suspended sediment consisted of 9-46 percent sand, 30-46 percent silt, and 20-56 percent clay. The median size of bed material was about 0.42 millimeter for three sets of measurements and about 0.18 millimeter for the other set. A dune bed form was present during all four datacollection periods. Data obtained on consecutive days indicate th at the turbulence constant can be computed from either streamflow-measurement notes or from vertical-velocity profiles. Constants computed from streamflow-measurement notes averaged 0.34 , and those from vertical-velocity profiles averaged 0.35 . The coefficients of vertical distribution of concertration for selected size ranges of suspended sands (expressed as $z_{1}$, the slope of the line relating the logarithms of concentration and a depth parameter) plotted against corresponding fall velocities indicate that on the average, the $z_{1}$ 's are proportional to about the 0.7 power of the fall velocity. The data also indicate that the relation of $z_{1}$ to fall velocity may vary with the mean velocity of flow.
\end{abstract}

\section{INTRODUC'TION}

Comprehensive data for computation of hydraulic and sediment parameters of the deep flow of large rivers are obtained only rarely. A small amount of such data was obtained for the Mississippi River at St. Louis during 1948-60 and was used by Jordan (1965) as the basis for his discussion of flow resistance, vertical distribution of velocity and suspended sediment, and bed-material discharge of the river at that site. The purpose of the present study was to obtain additional data from which selected hydraul: $c$ and sediment parameters could be computed for the deep flows of the Mississippi River at St. Louis. Four sets of data-each inc'uding water-surface slopes, computed energy gradients, vertical-velocity 
profiles, streamflow measurements, water temperature ${ }^{\sim}$ distribution of sediment in the vertical, and size distributions of suspended sediment and bed material-were obtained in April 1961, April and October 1962, and April 1963. From these data, solected hydraulic and sediment parameters were computed. A dotailed explanation of data-collection procedures and computation of the parameters are presented in this report; the data should help solve some problems relating to sediment transport by deep fows.

All the measurements were made in the river reach between mile 176.8 and mile 181.0 upstream from the mouth of the Ohio River (fig. 1). This reach is slightly curved and, except for being slightly wider between miles 177 and 179 than between miles 179 and 181, is of fairly uniform width. MacArthur Bridge, at mile 178.9, was the site of all velocity and water-tempersture measurements and of all sampling of suspended sedimert and bed material. Measurements of water-surface width and elevation were made at both ends of the reach and at selected intermediate cross sections. The St. Louis District of the U.S. Army Corps of Engineers cooperated in the study by establishing temporary bench marks at several places along the river edge and by operating a depth sounder to determine transverse and longitudinal profiles of the riverbed. During no set of measurements did the river stage fluctuate more than 3 feet; during the last set the range of fluctuation was slightly less than 0.5 foot.

\section{HYDRAULIC DATA}

Hydraulic data include streamflow measurements and point velocities obtained at mile 178.9, water-surface slopes, longitudinal and transverse profiles of the riverbed, and cross-section areas at miles 181.0, 178.9, and 176.8 or 177.1. Energy grad'onts were determined from water-surface elevations and computed velocity heads; turbulence constants were computed from streamflow measurement notes or from vertical velocity profiles.

\section{STREAMFLOW MEASUREMENTS AND CROSS-SECTICN AREAS}

Streamflow was measured on 3 days during the first datacollection period, on 2 days during each of the second and third periods, and on 3 days during the fourth period (table 1). Additional determinations of streamflow were made from ol $\times$ ervations of gage height and from stage-discharge relations for the river at Eads Bridge. The determination of shift adjustments to the rating curve was based on streamflow measurements and an assumed straight-line change of shift with time. Cross-section 


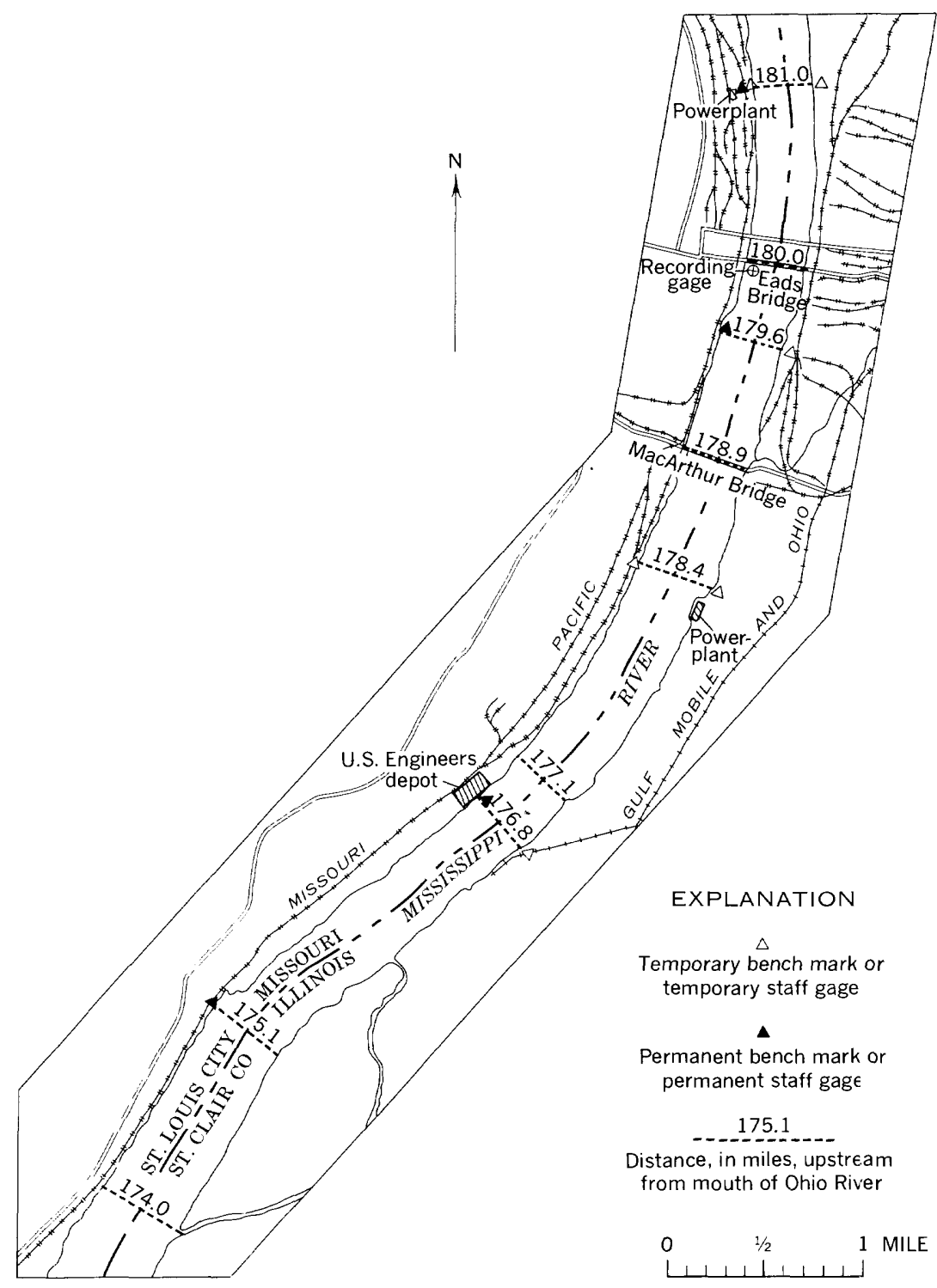

Figure 1.-Study reach of Mississippi River at St. Louis, Mo.

areas for intermediate times were determined by plotting areas from streamflow measurements against stage; areas determined from soundings obtained in connection with vertical-rolocity measurements were used to aid in interpolating the are?-stage relation when only two streamflow measurements were arailable for a data-collection period. 
TABLE 1.-Streamflow measurements from MacArthur Bridge, mile 178.9

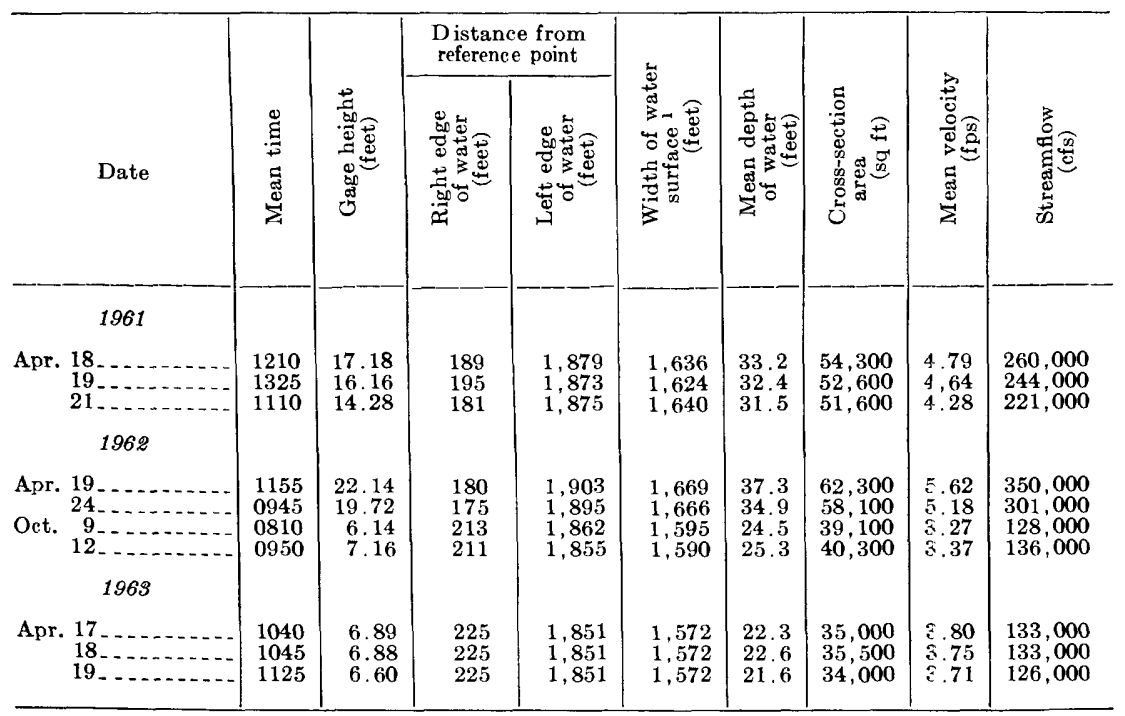

1 Difference between right and left edge of water minus width of bridge piers (54 ft).

Cross-section areas at other than mile 178.9 were obtained by means of a depth sounder mounted in a boat. A correctly oriented large-scale map affixed to a planetable was used for dotermining the positions of the boat, and on signal from the sounder operator, the sounder chart and map were marked simultaneously at about 10 points along each section traversed by the boat. Prcfiles of the riverbed, as determined from the soundings, are shown in figure 2. Cross-section areas determined from the sounded profiles were corrected to the common time of the water-surface-slope determinations by adding or subtracting the net change of gage height multiplied by the average water-surface width of tro sounded sections (table 2).

\section{WATER-SURFACE SLOPES}

Water-surface elevations used in slope observations were obtained from staff gages set near the water edge (fig. 1). Staff gages were established for each data-collection period, and levels were run from temporary bench marks to establish the elevation of the top of each staff. The water-surface elevation was determined by measuring from the top of the staff to the water surface. When wind, especially if moving generally in an upstream or downstream direction, caused waves that made deternination of the water-surface elevation difficult, two independent readings were 


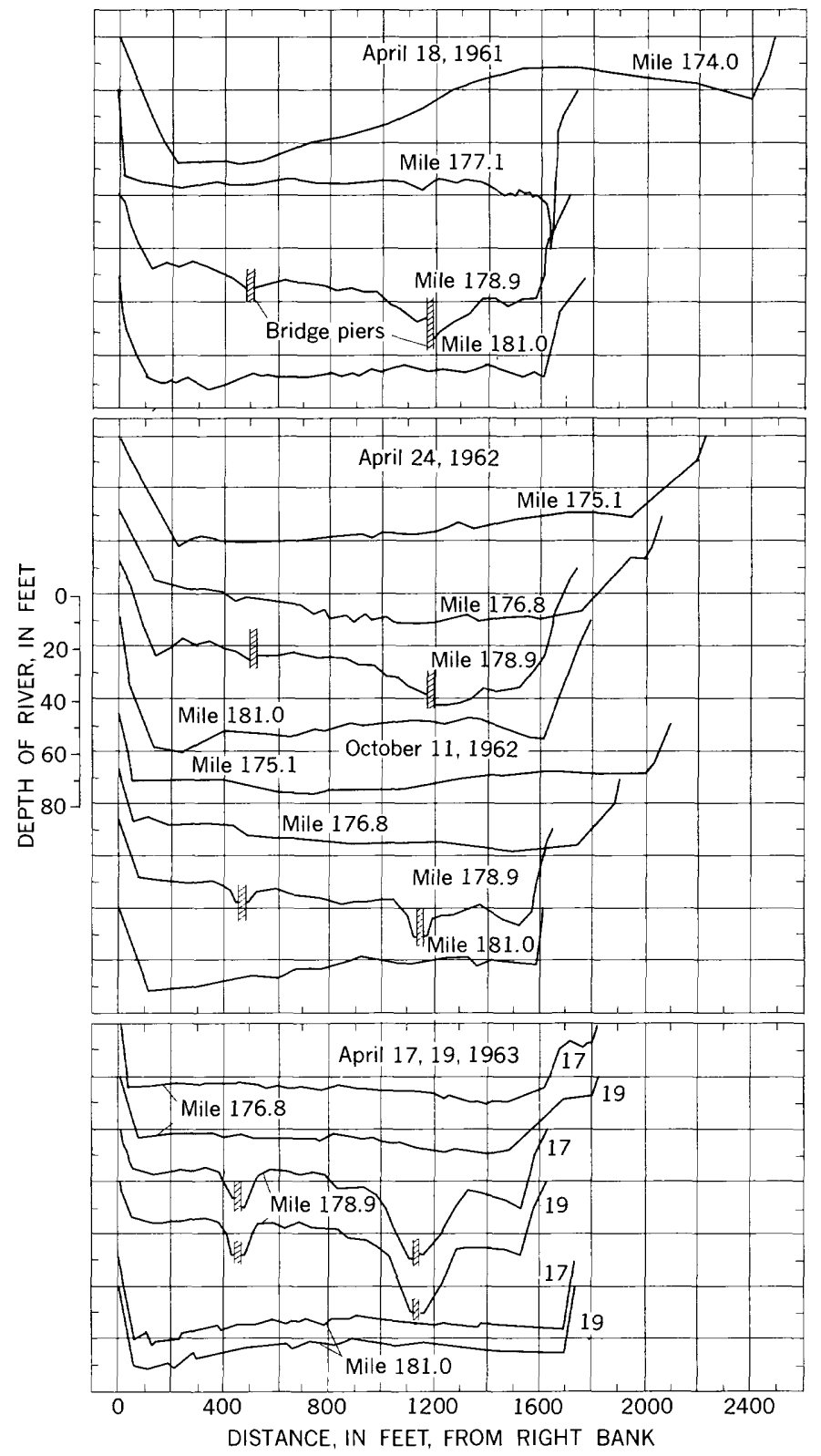

FIGURE 2.-Transverse profiles of riverbed. 
TABLE 2.-Cross-section data obtained with a depth sourder

\begin{tabular}{|c|c|c|c|c|c|}
\hline Date & $\begin{array}{l}\text { Location (miles } \\
\text { upstream from } \\
\text { Ohio River) }\end{array}$ & $\begin{array}{l}\text { Width } \\
\text { (feet) }\end{array}$ & $\begin{array}{l}\text { Mean } \\
\text { depth } \\
\text { (feet) }\end{array}$ & $\begin{array}{l}\text { Cross- } \\
\text { section } \\
\text { area } \\
\text { (sq ft) }\end{array}$ & $\begin{array}{l}\text { Mean } \\
\text { velocity } \\
\text { (fps) }\end{array}$ \\
\hline 1961 & & & & & \\
\hline Apr. 18 & $\begin{array}{l}181.0 \\
177.1\end{array}$ & $\begin{array}{l}1,760 \\
1,730\end{array}$ & $\begin{array}{l}34.2 \\
35.1\end{array}$ & $\begin{array}{l}60,220 \\
60,670\end{array}$ & $\begin{array}{l}4.37 \\
4.33\end{array}$ \\
\hline 1962 & & & & & \\
\hline Apr. 24 & $\begin{array}{l}181.0 \\
176.8\end{array}$ & $\begin{array}{l}1,780 \\
2,050\end{array}$ & $\begin{array}{l}39.1 \\
32.3\end{array}$ & $\begin{array}{l}69,700 \\
66,400\end{array}$ & $\begin{array}{l}4.30 \\
4.54\end{array}$ \\
\hline Oct. $11 \ldots \ldots$ & $\begin{array}{l}181.0 \\
176.8\end{array}$ & $\begin{array}{l}1,610 \\
1,900\end{array}$ & $\begin{array}{l}22.4 \\
22.1\end{array}$ & $\begin{array}{l}36,100 \\
42,000\end{array}$ & $\begin{array}{l}3.79 \\
3.26\end{array}$ \\
\hline 1963 & & & & & \\
\hline Apr. $17 \ldots \ldots$ & $\begin{array}{l}181.0 \\
176.8\end{array}$ & $\begin{array}{l}1,730 \\
1,820\end{array}$ & $\begin{array}{l}23.9 \\
22.8\end{array}$ & $\begin{array}{l}41,400 \\
41,400\end{array}$ & $\begin{array}{l}3.22 \\
3.21\end{array}$ \\
\hline $19 \ldots$ & $\begin{array}{l}181.0 \\
176.8\end{array}$ & $\begin{array}{l}1,730 \\
1,820\end{array}$ & $\begin{array}{l}23.0 \\
21.6\end{array}$ & $\begin{array}{l}39,850 \\
39,200\end{array}$ & $\begin{array}{l}3.18 \\
3.22\end{array}$ \\
\hline
\end{tabular}

obtained and averaged; the difference between the independent readings was commonly less than 0.05 foot. The water-surface elevations were plotted in the field as a check for errors; where apparently valid elevations seemed to be incorrect compared with other elevations, the staff on the opposite bank was reread as a check. Elevations of staff gages were redetermined whenever it seemed that the staff might have settled.

The water-surface elevation was generally slightly higher on the left side of the river than on the right. Curvature of the channel through most of the study reach may have caused the slight increase in water-surface elevation from right to left. At times, particularly in April 1963, the water-surface elevation on the right bank at mile 181.0 was somewhat inconsistent with the elevation on the left bank; apparently the elevation on the right bank is influenced by a large eddy created by release of powerplant cooling water returned to the river a short distance upstream.

Because the reading of the water-surface elevation at all the staff gages required 3-4 hours, the readings had to bo corrected to a common time so they could be used for determining instantaneous slopes of the water surface. The elevations were corrected to a common time from the change of stage per hour at the recording gage located at mile 180.0. To determine whether the changes in stage at the recording gage were representative of the entire reach during each data-collection period, hourly stage readings were obtained from a staff gage at the Corps of Engineers depot at mile 176.8. These readings indicated that the rate of change of 
stage at the recording gage was satisfactory for use along the entire reach for all the sets of data.

\section{ENERGY GRADIENTS}

The energy gradient was computed by adding a velocity head to the corrected water-surface elevations at miles 181.0, 178.9, and either 177.1 or 176.8 . The velocity head was computed from the equation

where

$$
\text { velocity head }=\alpha_{1} \frac{\bar{u}^{2}}{2 g},
$$

$\bar{u}$ is the mean velocity at the cross section,

$g$ is the acceleration of gravity, and

$\alpha_{1}$ is a correction factor.

The correction factor is approximated by the equation

$$
\alpha_{1}=\frac{\left(\frac{K_{a}{ }^{3}}{A_{a}{ }^{2}}+\frac{K_{b}{ }^{3}}{A_{b}{ }^{2}}+\ldots+\frac{K_{n}{ }^{3}}{A_{n}{ }^{2}}\right)}{\frac{(\Sigma K)^{3}}{(\Sigma A)^{2}}},
$$

where the subscripts denote subsections in the cross section and

$A$ is the area of the subsection, and

$K \quad$ is equal to $\frac{1.49}{n} A R^{2 / 3}$, where

$R$ is the hydraulic radius for which mean depth is substituted in these computations, and

$n$ is Manning's coefficient of roughness (Kindsvater and others, 1953).

However, as $n$ for the subsections is unknown, $n$ for the section is assumed to be constant and $\alpha$ is approximated by the equation

$$
\alpha_{1}=\frac{(\Sigma A)^{2}\left(A_{a} R_{a}^{2}+A_{b} R_{b}^{2}+\ldots+A_{n} R_{n}^{2}\right)}{\left(A_{a} R_{a}^{2 / 3}+A_{b} R_{b}^{2 / 3}+\ldots+A_{n} R_{n}^{2 / 3}\right)^{3}} .
$$

The water-surface slopes and the computed energy gredients (fig. 3) upstream from mile 178.9 (MacArthur Bridge) nay be either higher or lower than the slopes downstream from mile 178.9. The relation between the slopes upstream from the bridge and the slopes downstream from the bridge does not depend on a single variable such as streamflow; instead, it depends on several variables, of which streamflow and scour and fill in the reach are probably the most important. Jordan (1965, p. 29) conputed water-surface slopes from gages 4.4 miles upstream and 2.1 miles downstream from mile 178.9 and reported as follows: "During 1950-53, the slopes changed widely from time to time, but the 

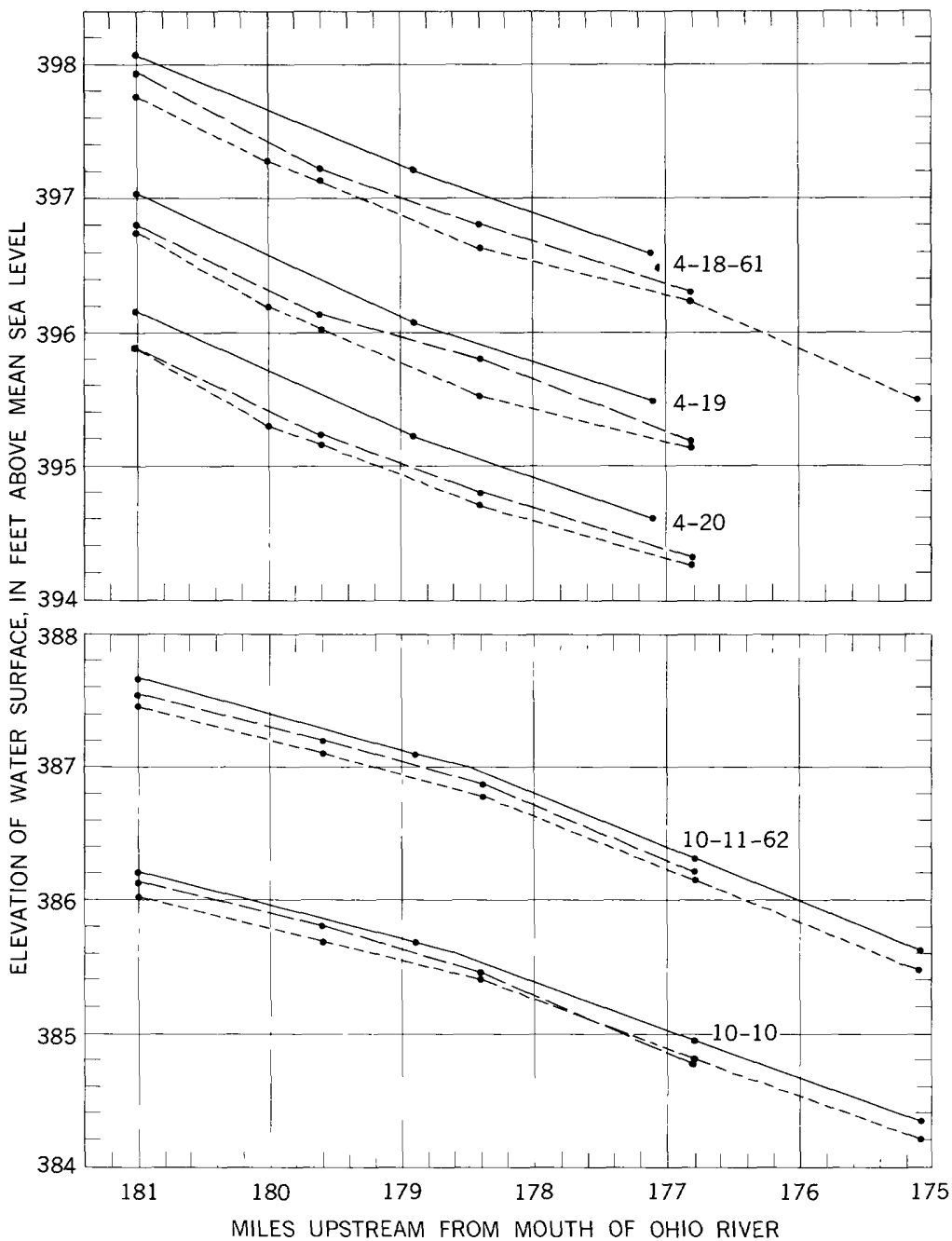

EXPLANATION

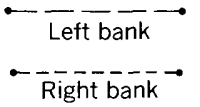

Water-surface elevation

Water-surface elevation at right bank plus velocity head

FIGURE 3.-Longitudinal water-surface and energy prof:les. 


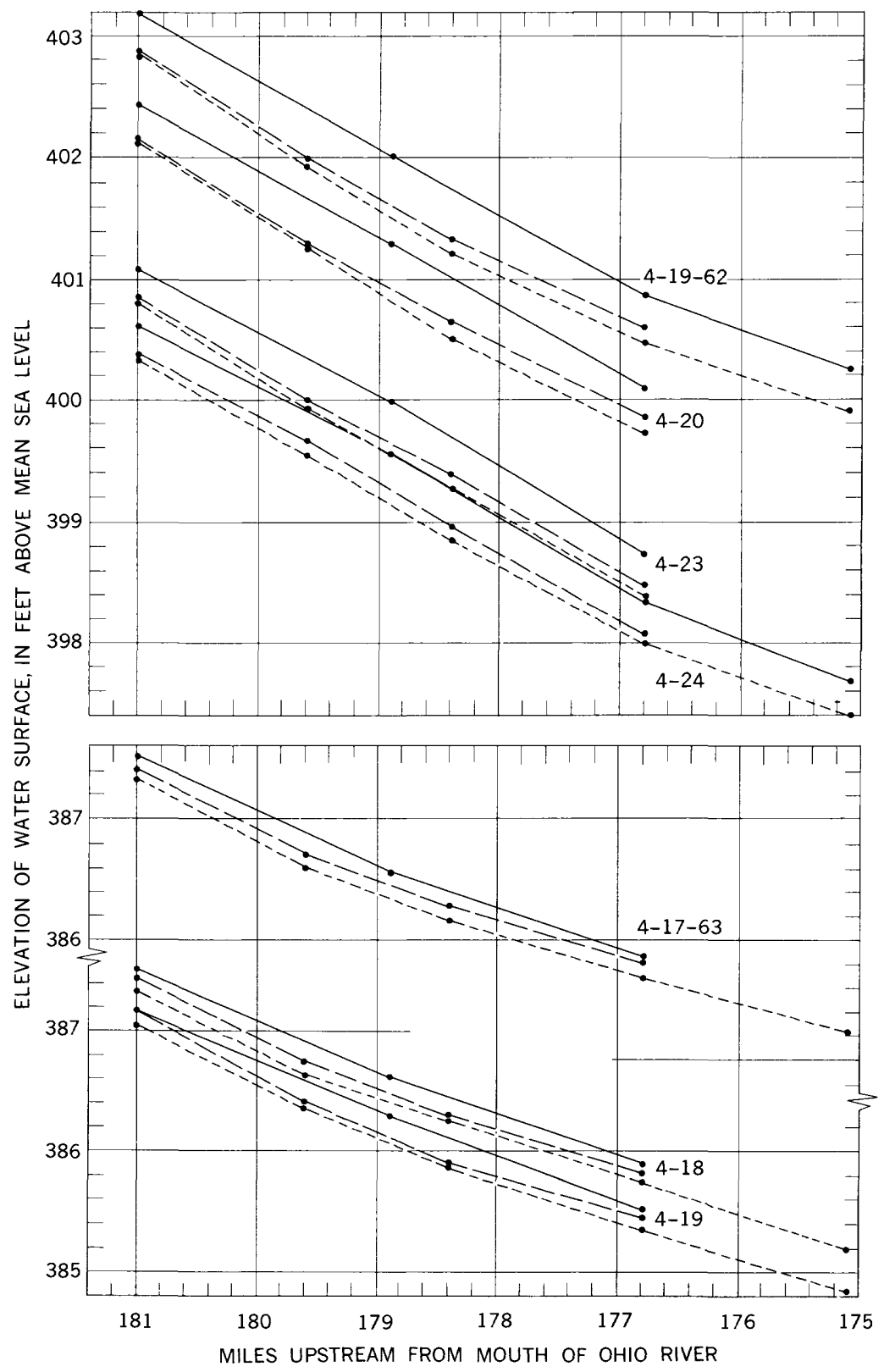

FIGURE 3.-Continued. 
slopes upstream and downstream from the bridge wer? generally about the same at any given time. The slopes in the upstream reach tended to be slightly steeper than the slopes in the downstream reach. During 1954-59, the slopes were fairly constant, but those in the upstream reach were consistently steeper than those in the downstream reach; in 1956-57, the slopes upstream were about twice as steep as those downstream."

\section{LONGITUDINAL BED PROFILES}

Using a depth sounder, the Corps of Engineers, St. Louis District, determined the longitudinal bed profile at abcut quarter points of width for each set of data. (See figs. 4 ard 5.) The range lines shown on the charts are not necessarily the same range lines for the different sets of data, but the approximate locations of the mile markers are shown to aid comparison of the profiles; streamflow is in the direction of decreasing mileage. Trese profiles were obtained to provide data to aid in studies of tre relations between bed forms and roughness and in studies of depth effect on bed forms. Also, the profiles were obtained to determine changes in bed configuration that might be related to the breaks in water-surface slopes (fig. 3) and to determine possible causes for the breaks in slope. The left pier of MacArthur B-idge (mile 178.9) causes a definite scour hole and an area of deposition downstream from the bridge on the left side of the channel; the causes for changes in bed elevation and changes in size and shape of dunes at other places in the reach are obscure.

The longitudinal profiles do not show any changes in the bed that might be related, either as to cause or effect, to the breaks in water-surface slopes. The major breaks in water-surface slopes, when they occur, are in the vicinity of miles 179 and 177 . There are two bridges in the study reach upstream from mile 179, but there is no bridge in the vicinity of mile 177 ; however, watersurface width (fig. 1) is somewhat greater between miles 179 and 177 than either upstream or downstream from that reach. A map of a detailed survey of the Mississippi River in the vicinity of St. Louis made by the Corps of Engineers in 1956 showed the reach between miles 179 and 177 to be about 200 feet wider than the reaches upstream and downstream. The location of the breaks in slope seems more likely to be related to changes in channel width and cross-sectional area than to location of bridges.

\section{VERTICAL DISTRIBUTION OF VELOCITY}

Point velocities for definition of vertical-velocity profiles (pl. 1) were obtained at about 15 verticals on 1 or 2 days of each data- 


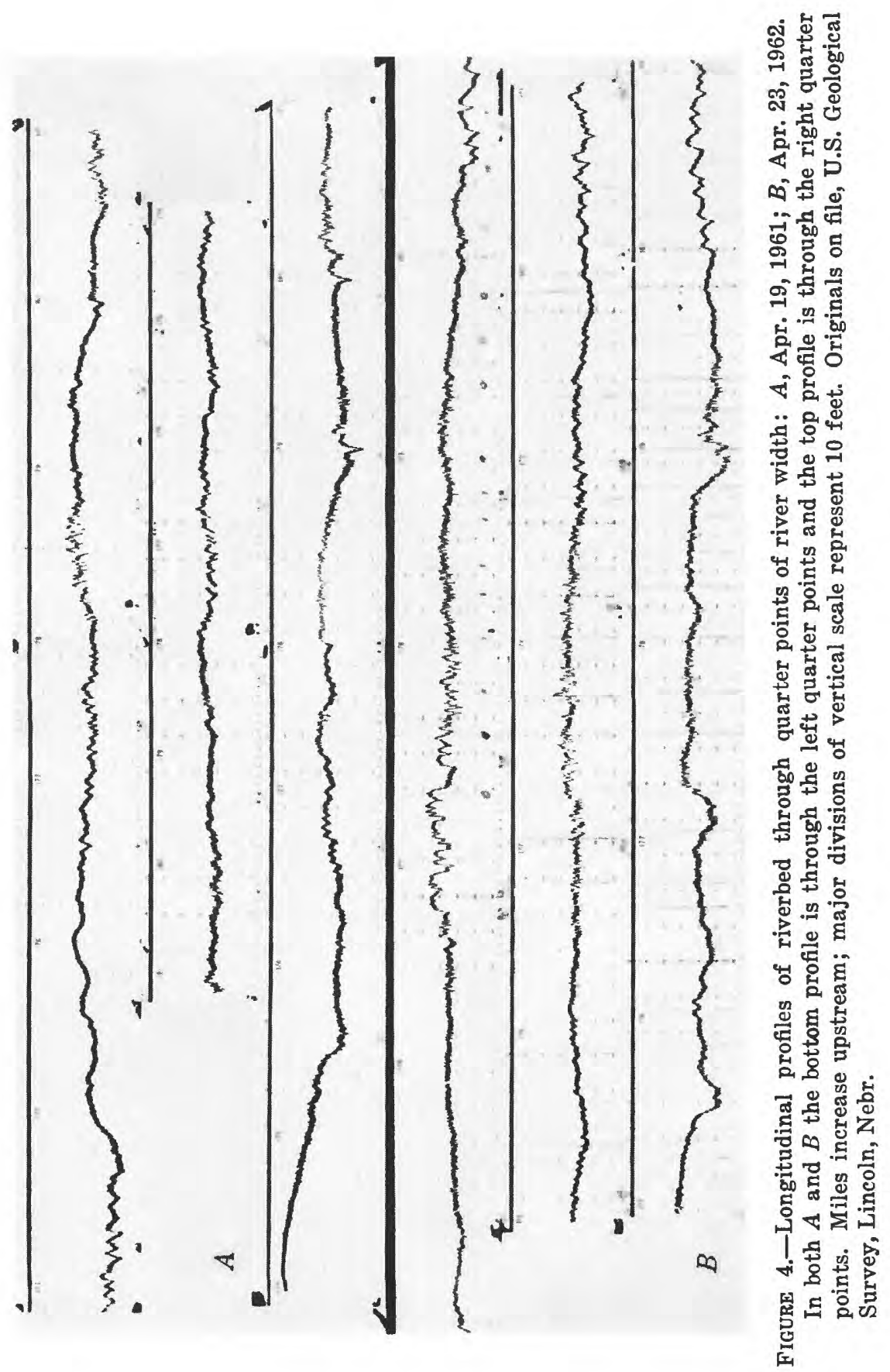


J12 CONTRIBUTIONS TO THE HYDROLOGY OF THE UNITED STATES

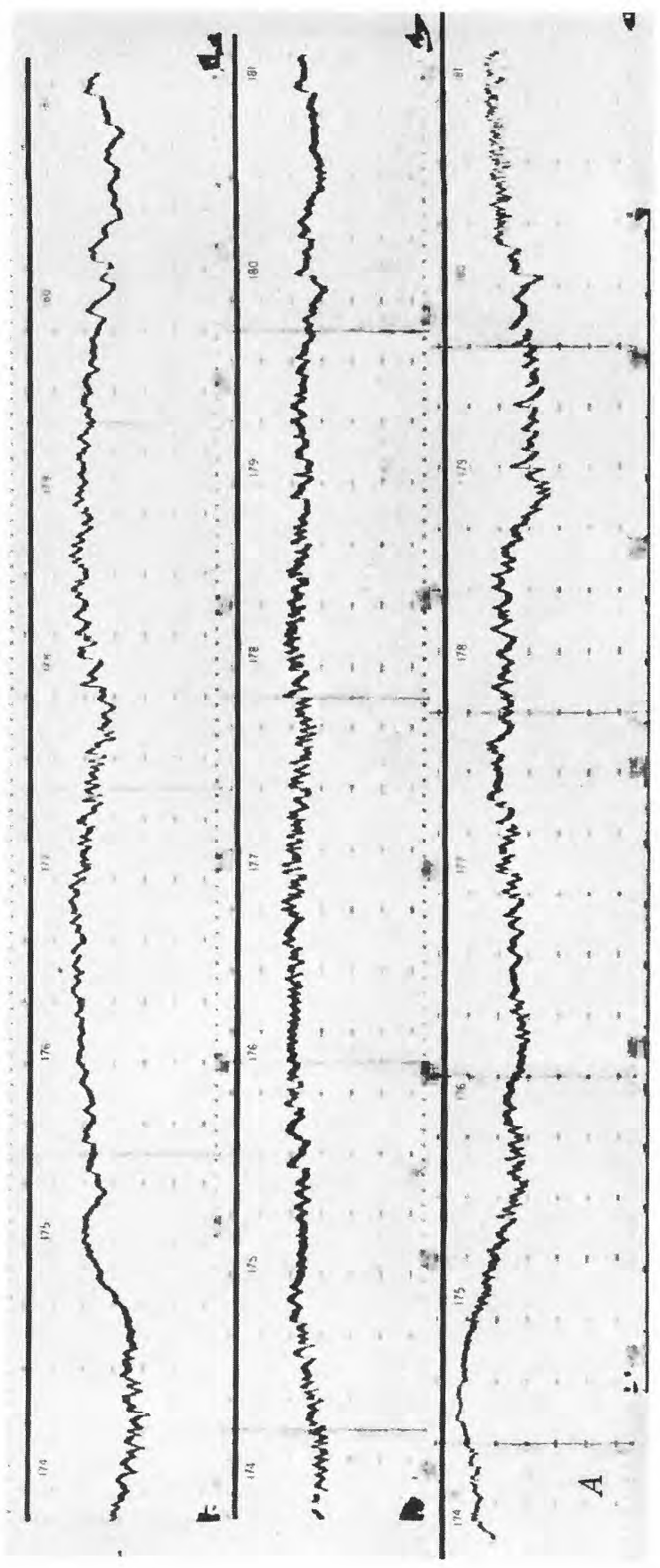




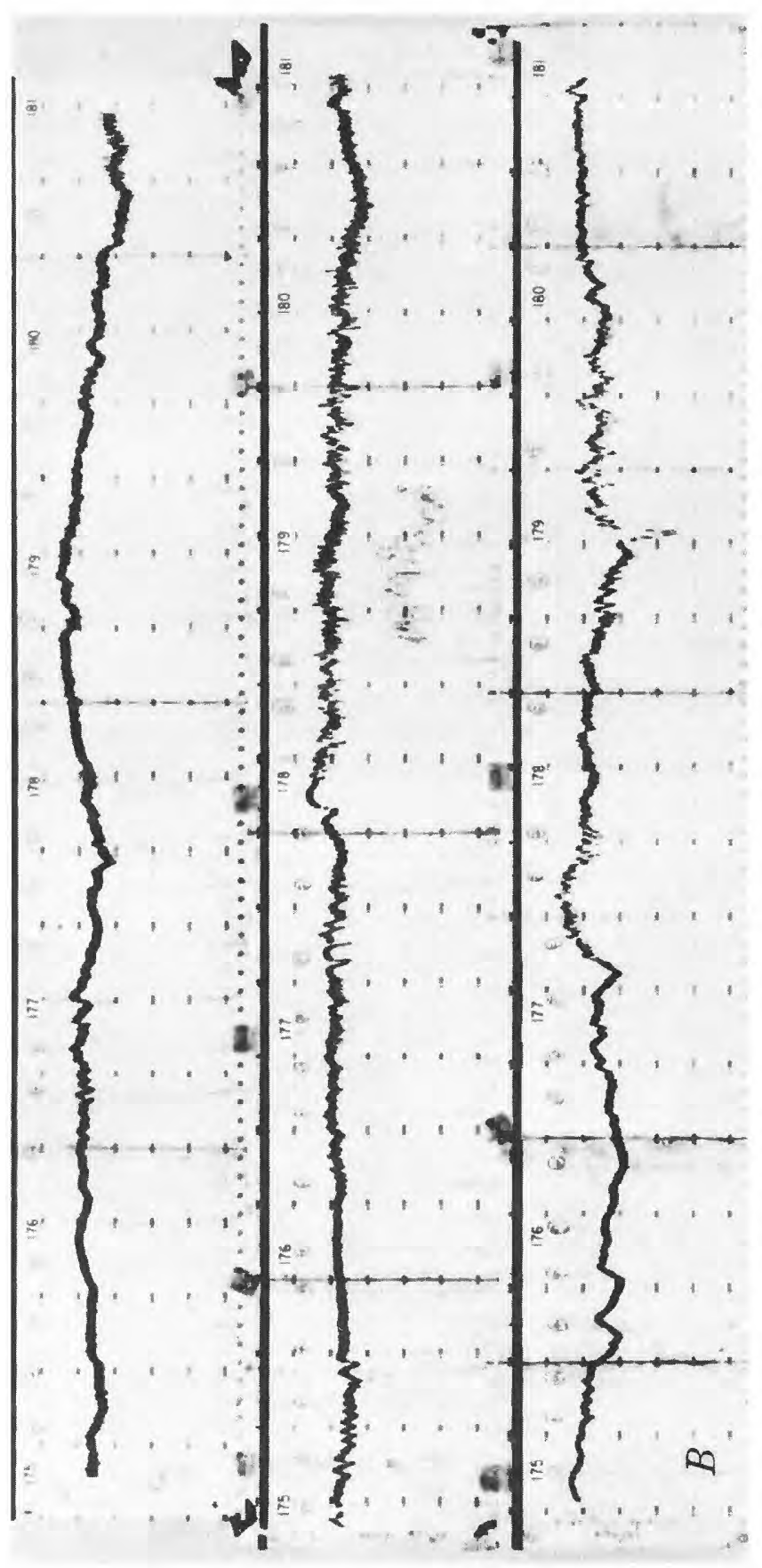
悹焉

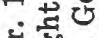
品 运 ํํ욜 윽 형 댄 ․․․․ डั ॠ虽 동 믕 $+0$ 욜 달 넝 행 要

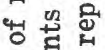
녈 옹 क्षे म 可焉 焉焉

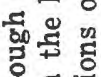
홓 우 웅 曹 농 政 넝 茖 怘 五总芯 我艺 등

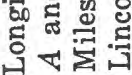
个这 20. 若热 国察总 点 
collection period except in April 1963, during which verticalvelocity profiles were obtained only at the 6 verticals used for point sampling. The point-velocity measurements used in preparing the vertical-velocity profiles are presented in table 3. All point velocities were plotted against the logarithm of distance above the bed at the time they were obtained, and any velocities that appeared to be in error (except those obtained to determine short- and long-term changes in the vertical velocity on April 18 and 19) were rerun immediately. On April 18, three velocity profiles were obtained in succession at about 15-minute intervals at station 825 , and the three profiles were repeated about 3 hours later. A barge had just passed near the station when the first set of velocity profiles was begun, and it definitely affected the profile at 0905 and probably affected the profile at 0920 . The three profiles of the set that was started at 1200 show some variations, as do the profiles of the sets at station 1250 on April 19 beginning at 0935 and 1235 . The average slope of the first set of three profiles at station 1250 was considerably different from the slope of the second set of three profiles at the same station. The differences between the average slopes of the sets are caused by shifting of the bed as dunes move through the section.

TABLE 3.-Measured velocity, in feet per second, at selected points above riverbed, April 1961, April and October 1962, and April 1963

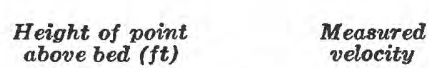

\section{1}

Station 300

Apr. 20; total depth, $24.3 \mathrm{ft}$; mean time, 1715 ; gage height, $14.96 \mathrm{ft}$

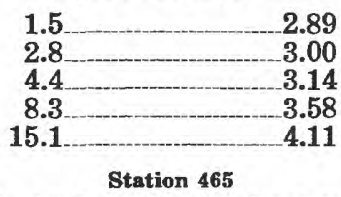

Apr. 20; total depth, $24.5 \mathrm{ft}$; mean time, 1700 ; gage height, $14.99 \mathrm{ft}$

\begin{tabular}{lr}
1.5 & 0.86 \\
\hline 2 & .93 \\
2.8 & 2.16 \\
3.4 & 3.07 \\
4.4 & 3.27 \\
6.1 & 3.76 \\
8.3 & 4.14 \\
11.3 & 4.47 \\
15.2 & 4.90 \\
& -9.36 \\
20.8 & 4.88
\end{tabular}

\section{Height of point Measured above bed ( $f t)$}

Station 625

Apr. 20; total depth, $31.4 \mathrm{ft}$

$\begin{array}{rr}1.5 & 1.80 \\ 2.9 & 2.62 \\ 5.3 & 3.04 \\ 11.2 & 3.16 \\ 19.1 & 2.91 \\ & 4.43 \\ & \text { Station } 800\end{array}$

Apr. 20 ; total depth, $30.8 \mathrm{ft}$

$\begin{array}{rr} & 2.80 \\ 1.5 & 3.27 \\ 2.1 & 2.94 \\ 2.9 & 3.37 \\ 3.7 & 3.79 \\ 5.2 & 4.08 \\ 7.1 & 4.11 \\ & 3.72 \\ 9.9 & 4.43 \\ 13.5 & 4.44 \\ 18.8 & -4.58 \\ & 4.24 \\ 26.2 & 5.13\end{array}$


TABLE 3.-Measured velocity, in feet per second, at selected points above riverbed, April 1961, April and October 1962, and April 1963-Continued

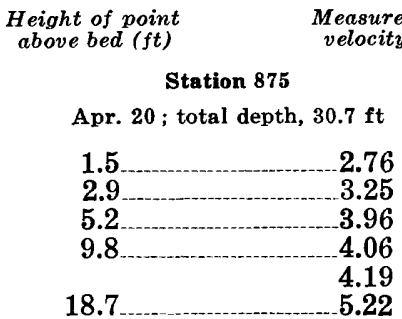

Station 975

Apr. 20; total depth, $33.1 \mathrm{ft}$

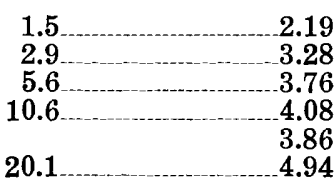

Station 1050

Apr. 20 ; total depth, $35.4 \mathrm{ft}$

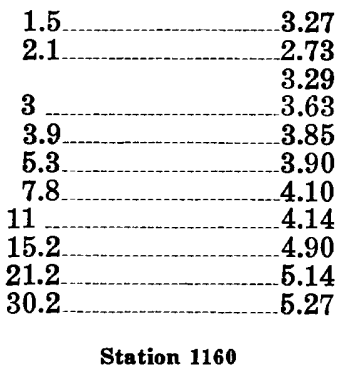

Apr. 20 ; total depth, $37.3 \mathrm{ft}$

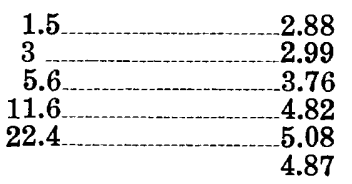

Station 1250

Apr. 20 ; total depth, $38.9 \mathrm{ft}$

$\begin{array}{rr}1.5 & 2.35 \\ 2.1 & 2.91 \\ 3 & 2.28 \\ & 2.78 \\ 4.3 & 3.72 \\ 5.8 & 4.90 \\ 8.6 & 2.68 \\ & 4.86 \\ 12 & 4.64 \\ 16.7 & 5.00 \\ 23.4 & 5.35 \\ 33.1 & 5.72\end{array}$

\section{Height of point above bed ( $f t$ ) \\ Measured velocity \\ Station 1300}

Apr. 20 ; total depth, $45.5 \mathrm{f}^{*}$.

$\begin{array}{rr}1.5 & 2.86 \\ 3.2 & 3.47 \\ 5.5 & 3.40 \\ 11.8 & 3.76 \\ 25.2 & 4.54 \\ & 5.30\end{array}$

Station 1400

Apr. 20 ; total depth, $47.4 \mathrm{f}^{4}$.

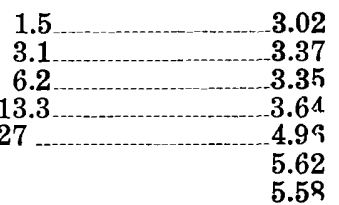

Station 1440

Apr. 19 ; total depth, $48.7 \mathrm{f}$ :

$\begin{array}{rr}1.5 & 2.71 \\ 2.2 & 3.07 \\ 3.1 & 3.2 \\ 4.6 & 3.70 \\ & 3.10 \\ 6.3 & 3.32 \\ & 3.33 \\ 9.2 & 3.8 ? \\ 13.6 & 4.19 \\ 19.5 & 5.13 \\ & 5.40 \\ 27.8 & 5.45 \\ 39.9 & 6.3 ?\end{array}$

Apr. 20 ; total depth, 47.2 f

$\begin{array}{rr}1.5 & 3.22 \\ 2.2 & 3.77 \\ 3.1 & 3.34 \\ & 3.19 \\ 4.4 & 3.27 \\ 6.1 & 3.79 \\ 8.9 & 4.07 \\ 13.2 & 3.75 \\ 18.9 & 4.87 \\ 26.9 & 5.04 \\ 38.7 & 5.62 \\ & 5.45 \\ & 5.7 ?\end{array}$

Station 1515

Apr. 18; total depth, $41.7 \mathrm{ft}$

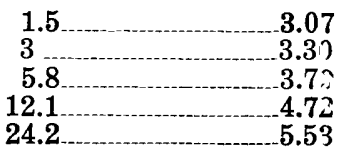




\section{J16 CONTRIBUTIONS TO THE HYDROLOGY OF THE UNITED STATES}

TABLE 3.-Measured velocity, in feet per second, at selected points above riverbed, April 1961, April and October 1962, and April 1963-Continued

Height of point above bed $(f t)$

\section{Measured} velocity

Station 1525

Apr. 20 ; total depth, $40.3 \mathrm{ft}$; mean time, 1300 ; gage height, $15.20 \mathrm{ft}$

$\begin{array}{r}1.5 \\ 3 \\ 5.6\end{array}-\begin{array}{r}1.46 \\ 11.7 \\ 23.4\end{array}$

Station 1565

Apr. 18 ; total depth, $39.0 \mathrm{ft}$; mean time, 1700 ; gage height, $16.90 \mathrm{ft}$

$\begin{array}{rr}1.5 & 4.12 \\ 3 & 4.29 \\ 5.8 & 4.64 \\ 12.1 & 4.29 \\ 23.2 & 5.26 \\ & -.5 .76\end{array}$

Station 1590

Apr. 18; total depth, $38.3 \mathrm{ft}$; mean time, 1600 ; gage height, $16.95 \mathrm{ft}$

$\begin{array}{rr}1.5 & 4.29 \\ & 4.32 \\ 2.1 & 4.27 \\ 3 & 4.68 \\ 4.2 & 4.18 \\ & 4.35 \\ 5.8 & 5.04 \\ 8.4 & 4.99 \\ 11.9 & 5.40 \\ 16.5 & 5.67 \\ 23 & 5.99 \\ 32.6 & 5.90\end{array}$

Apr. 19; total depth, $41.0 \mathrm{ft}$; mean time, 1600 ; gage height, $16.08 \mathrm{ft}$

$\begin{array}{rr}1.5 & 2.87 \\ 2.1 & 3.09 \\ 3 & 3.07 \\ & 3.80 \\ 4.1 & 3.96 \\ & 3.37 \\ 5.7 & 3.91 \\ 8.2 & 4.50 \\ 11.9 & -5.04 \\ 16.8 & 5.36 \\ 23.8 & 5.72 \\ 33.6 & 5.99\end{array}$

Apr. 20 ; total depth, $38.8 \mathrm{ft}$

\begin{tabular}{rr}
1.5 & 4.07 \\
2.1 & 4.07 \\
3 & 4.54 \\
4.3 & 3.75 \\
& 4.39 \\
5.8 & 4.18 \\
& 4.39 \\
8.5 & 4.51 \\
12 & 4.91 \\
\hline & 4.91
\end{tabular}

\section{Height of point above bed $(f t)$ \\ Measured \\ velocity}

Station 1590-Cont'nued

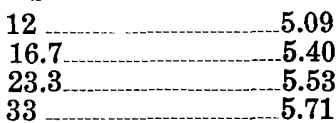

Station 1670

Apr. 20 ; total depth, $37.3 \mathrm{ft}$

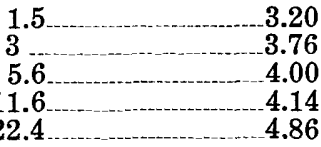

Station 1750

Apr. 20 ; total depth, $35.9 \mathrm{ft}$; mean time 1200 ; gage height, $15.25 \mathrm{ft}$

$\begin{array}{rr}1.5 & 1.89 \\ 3 & 2.40 \\ 5.4 & 2.33 \\ & 2.15 \\ 11.1 & 2.93 \\ 21.6 & 3.34\end{array}$

Apr. 18; total depth, $30.9 \mathrm{ft}$; mean time, 1530 ; gage height, $16.96 \mathrm{ft}$

$\begin{array}{rr}1.5 & 1.63 \\ 2.9 & 1.93 \\ 5.3 & 2.24 \\ 9.9 & 2.39 \\ 18.8 & 2.62\end{array}$

\section{2}

Station 300

Apr. 20: total depth, 35.1 ft: mean time 1620 ; gage height, $21.31 \mathrm{ft}$
1.5
3.47
2.5
3.12
4.1
4.03
6.7
4.32
11
18
4.70
30
5.23
5.44

Apr. 23 ; total depth, $33.7 \mathrm{ft}$; mean time 1300 ; gage height, $20.13 \mathrm{ft}$
1.5 .
2.4
2.82
3.9
3.40
$6.2 \quad 3.78$
$10 \ldots$
16

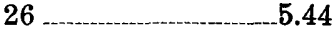
Station 400

Apr. 20 ; total depth, $28.7 \mathrm{ft}$

1.5
2.4


TABLE 3.-Measured velocity, in feet per second, at selected points above riverbed, April 1961, April and October 1962, and April 1963-Continued

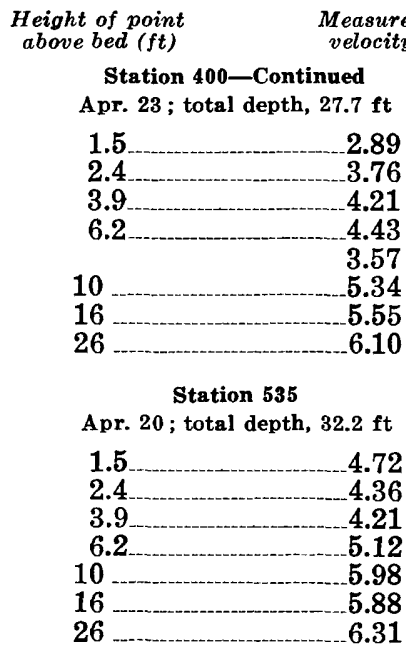

Apr. 23 ; total depth, $30.1 \mathrm{ft}$

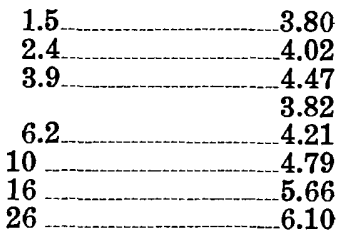

Station 625

Apr. 20 ; total depth, $36.4 \mathrm{ft}$

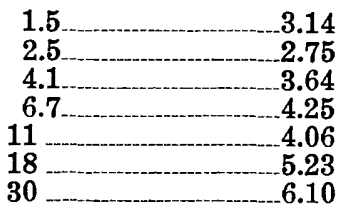

Apr. 23; total depth, $33.8 \mathrm{ft}$

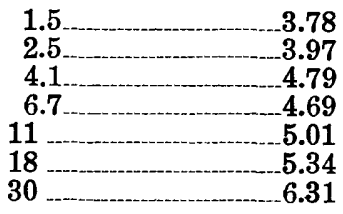

Station 725

Apr. 20 ; total depth, $35.2 \mathrm{ft}$

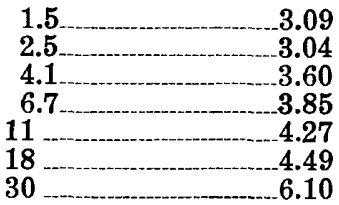

Height of point
above bed ( $f t)$

Station 725-Continued

Apr. 23 ; total depth, $34.2 \mathrm{ft}$

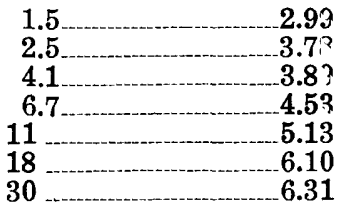

Station 900

Apr. 20 ; total depth, $36.0 \mathrm{ft}$

1.5
2.5
4.2

Apr. 23 ; total depth, 35.1 f

1.5
2.5
4.1 $\begin{array}{r}2.79 \\ 6.7 \\ 11\end{array}$

Station 985

Apr. 20 ; total depth, $36.5 \mathrm{f}$ :

1.5
2.5
4.2
7 $\quad \begin{array}{r}3.02 \\ 12 \\ 20 \\ 33\end{array}$

Apr. 23 ; total depth, $35.8 \mathrm{f}$ :

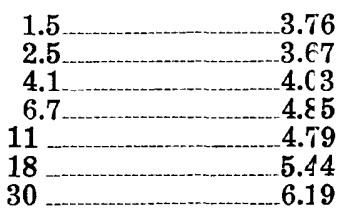

Station 1100

Apr. 20 ; total depth, $38.8 \mathrm{ft}$

1.5
2.5
4.1
4.7
6.79
11
18
18
30




\section{J18 CONTRIBUTIONS TO THE HYDROLOGY OF THE UNITED STATES}

TABLE 3.-Measured velocity, in feet per second, at selected points above riverbed, April 1961, April and October 1962, and April 1963-Continued

$\begin{array}{rr}\begin{array}{c}\text { Height of point } \\ \text { above bed (ft) }\end{array} & \begin{array}{r}\text { Measure } \\ \text { velocity }\end{array} \\ \text { Station } 1100 \text { Continued } \\ \text { Apr. } 23 \text {; total depth, } 37.2 \mathrm{ft} \\ 1.5\end{array}$

Apr. 23 ; total depth, $41.6 \mathrm{ft}$

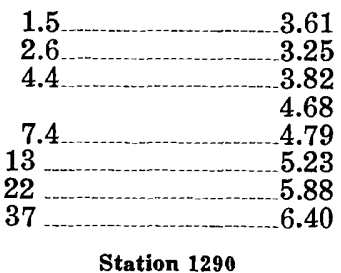

Apr. 20 ; total depth, $49.5 \mathrm{ft}$

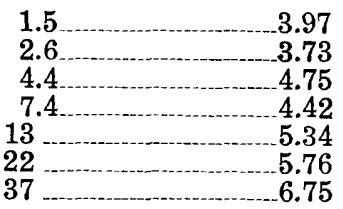

Apr. 23 ; total depth, $45.6 \mathrm{ft}$

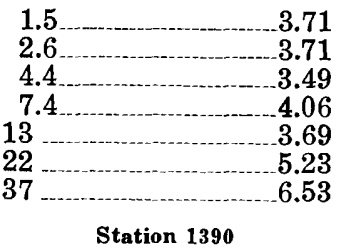

Apr. 20 ; total depth, $54.3 \mathrm{ft}$

\begin{tabular}{|c|}
\hline $\begin{array}{l}1.5 \\
2.6 \\
4.5 \\
7.9\end{array}$ \\
\hline
\end{tabular}

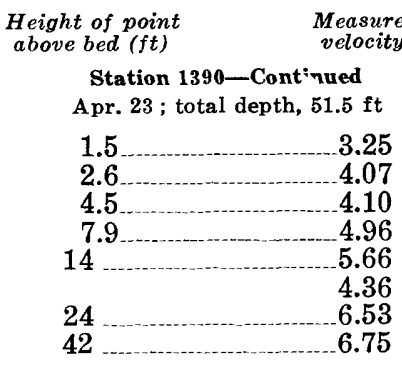

Station 1450

Apr. 20 ; total depth, $52.5 \mathrm{ft}$

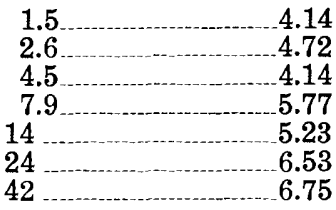

Apr. 23 ; total depth, $51.2 \mathrm{ft}$

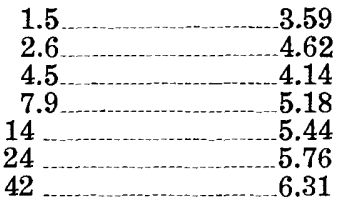

Station 1585

Apr. 20 ; total depth, $49.5 \mathrm{ft}$

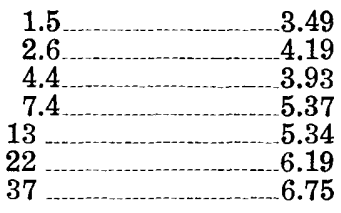

Apr. 23 ; total depth, $47.5 \mathrm{ft}$

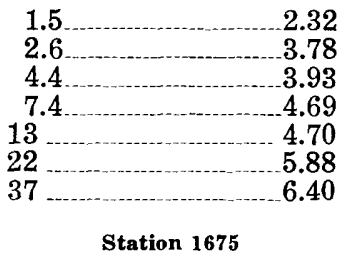

Apr. 20 ; total depth, $49.4 \mathrm{ft}$

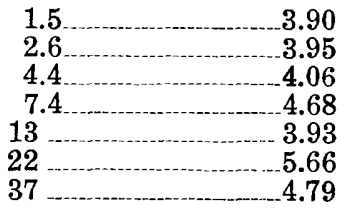


TABLE 3.-Measured velocity, in feet per second, at selected points above riverbed, April 1961, April and October 1962, and April 1963-Continued

\section{Height of point above bed ( $f t$ )

Measured
velocity

Station 1675-Continued

Apr. 23 ; total depth, $45.9 \mathrm{ft}$

\begin{tabular}{rr}
1.5 & 3.80 \\
2.6 & 3.78 \\
4.5 & 3.69 \\
7.9 & 4.47 \\
14 & 4.21 \\
24 & 4.58 \\
42 & 5.55 \\
\hline & 5.76
\end{tabular}

Station 1725

Apr. 20; total depth, $44.0 \mathrm{ft}$; mean time, 0930 ; gage height, $21.48 \mathrm{ft}$

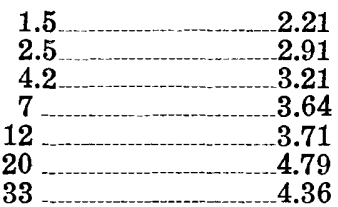

Apr. 23 ; total depth, $40.8 \mathrm{ft}$; mean time, 0900 ; gage height, $20.19 \mathrm{ft}$

\begin{tabular}{rr}
1.5 & 2.23 \\
2.6 & 2.70 \\
4.4 & 3.89 \\
7.4 & 3.60 \\
13 & 4.36 \\
22 & 4.23 \\
37 & 3.98 \\
\hline & 4.40
\end{tabular}

\section{Station 375}

Oct. 10; total depth, $18.3 \mathrm{ft}$; mean time, 0940 ; gage height, $5.80 \mathrm{ft}$

\begin{tabular}{|c|c|}
\hline 15 & 159 \\
\hline 2.2 & 1.8 \\
\hline 3.3 & 2.0 \\
\hline 4.9 & 2.1 \\
\hline 7.2 & 2.3 \\
\hline 10.8 & 2.4 \\
\hline
\end{tabular}

Oct. 11; total depth, $20.1 \mathrm{ft}$; mean time, 0925 ; gage height, $7.11 \mathrm{ft}$

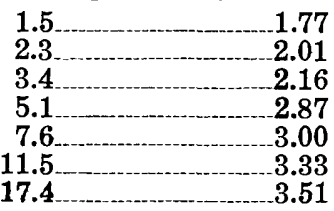

Station 525

Oct. 10; total depth, $18.9 \mathrm{ft}$

$\begin{array}{rr}1.5 & 2.38 \\ 2.2 & 2.55 \\ 3.3 & 2.75 \\ & 3.12 \\ 5 & 3.30 \\ 7.4 & 3.57\end{array}$

\section{Height of point above bed ( $\mathrm{ft}$ ) \\ Measured velocity}

Station 525-Continued

11
16.4
2.75
$3.6 €$

Oct. 11 ; total depth, $20.0 \mathrm{ft}$

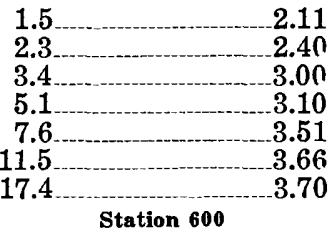

Oct. 10 ; total depth, $19.0 \mathrm{ft}$

$\begin{array}{r}1.5 \\ 2.2 \\ 3.3\end{array}-\begin{array}{r}2.74 \\ 5\end{array}$

Oct. 11 ; total depth, $19.4 \mathrm{ft}$

1.5

$2.3 \quad 2.8 ?$

$3.4 \quad 3.09$

5 - 3.25

7.4

11.1

16.6

3.9 )

Station 775

Oct. 10 ; total depth, $20.9 \mathrm{ft}$

$\begin{array}{rr}1.5 & 2.59 \\ 2.3 & 2.75 \\ 3.4 & 2.59 \\ & 3.07 \\ 5.2 & 3.0 ? \\ 7.8 & 3.14 \\ 12 & 3.15 \\ 18 & 3.7\end{array}$

Oct. 11 ; total depth, $22.9 \mathrm{ft}$

1.5
2.3
3.5
5.3
8.2
12.8
19.8

Station 900

Oct. 10 ; total depth, $23.6 \mathrm{f}$ -

$\begin{array}{rr}1.5 & 2.59 \\ 2.3 & 2.69 \\ 3.5 & 3.14 \\ 5.5 & 3.37 \\ 8.4 & 3.45 \\ 13.2 & 3.3^{\circ} \\ & 3.25 \\ 20.3 & 3.73\end{array}$




\section{J20 CONTRIBUTIONS TO THE HYDROLOGY OF THE UNITED STATES}

TABLE 3.-Measured velocity, in feet per second, at selected points above riverbed, April 1961, A pril and October 1962, and April 1963-Continued

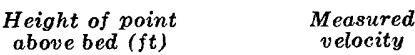

Station 900-Continued

Oct. 11; total depth, $25.2 \mathrm{ft}$

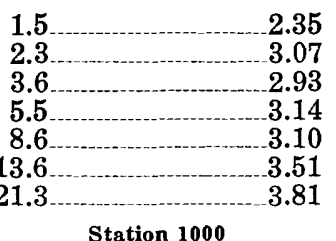

Oct. 10 ; total depth, $25.2 \mathrm{ft}$

1.5
2.3
3.6
5.7
8.8
13.9

Oct. 11; total depth, $26.9 \mathrm{ft}$

$\begin{array}{rr}1.5 & 3.04 \\ 2.3 & 3.00 \\ 3.6 & 3.07 \\ 5.7 & 2.75 \\ 9 & 3.03 \\ 14.4 & 3.20 \\ 22.9 & 3.99\end{array}$

Station 1100

Oct. 10 ; total depth, $25.9 \mathrm{ft}$

\begin{tabular}{rr}
1.5 & 2.56 \\
2.4 & 2.62 \\
3.7 & 2.78 \\
5.8 & 3.30 \\
9 & 3.14 \\
9 & 3.46 \\
14.2 & 3.78 \\
22.1 & 4.05 \\
\hline
\end{tabular}

Oct. 11 ; total depth, $27.6 \mathrm{ft}$

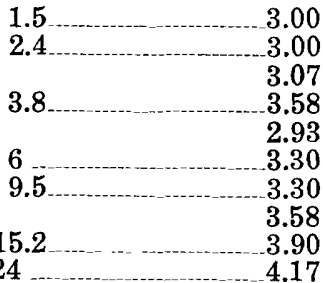

Station 1160

Oct. 10 ; total depth, $27.1 \mathrm{ft}$

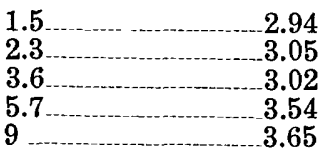

Height of point above bed ( $f t$ )

\section{Measured} velocity

\section{Station 1160-Continued}

14.4
22.9

3.84

4.11

Oct. 11 ; total depth, $28.4 \mathrm{ft}$

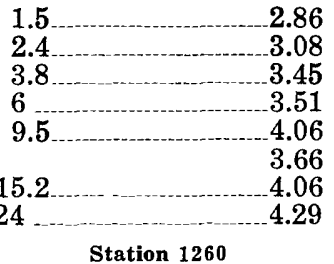

Oct. 10 ; total depth, $24.5 \mathrm{ft}$

1.5

2.62

2.3

3.07

3.6

3.73

5.5

3.86

8.5

4.27

13.4

4.30

20.9

4.27

4.27

4.38

Oct. 11 ; total depth, $27.2 \mathrm{ft}$

1.5

2.87

2.3

3.51

3.6

3.58 3.51

5.7

4.08

4.17

9

14.4

4.27

22.9

4.38

4.76

Station 1300

Oct. 10 ; total depth, $32.4 \mathrm{ft}$

1.5

1.92

2.5

2.06

4.1 .

2.62

6.9

3.07

11.3

3.82

3.73

19 3.81

31.7

4.17

Oct. 11; total depth, $33.7 \mathrm{ft}$

1.5

2.6

2.69

4.3

2.56

7.3

3.07

12.2

20.9

3.81

28.4

\section{Station 1410}

Oct. 10 ; total depth, $33.6 \mathrm{ft}$

1.5 .

2.6 
TABLE 3.-Measured velocity, in feet per second, at selected points above riverbed, April 1961, April and October 1962, and April 1963-Continued

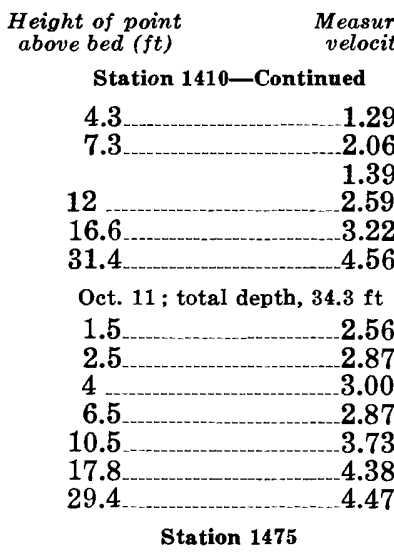

Oct. 10 ; total depth, $31.0 \mathrm{ft}$

1.5
2.4
3.9

Oct. 11 ; total depth, $32.5 \mathrm{ft}$

$\begin{array}{rr}1.5 & 3.00 \\ & 2.51 \\ 2.4 & 3.00 \\ & 2.69 \\ 3.8 & 2.93 \\ 6.3 & 2.75 \\ & 3.22 \\ 10.2 & 3.81 \\ 16.7 & 4.38 \\ & 3.90 \\ 27.3 & 4.66\end{array}$

Station 15501

Oct. 10 ; total depth, $27.9 \mathrm{ft}$

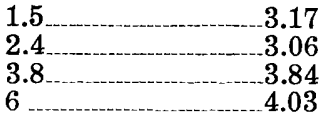

Oct. 11 ; total depth, $29.7 \mathrm{ft}$

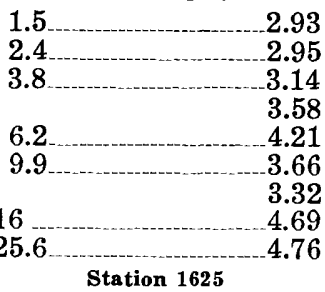

Oct. 11 ; total depth, $32.5 \mathrm{ft}$

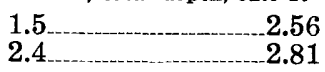

1 Station 1550 not completed owing to equipment breakdown.

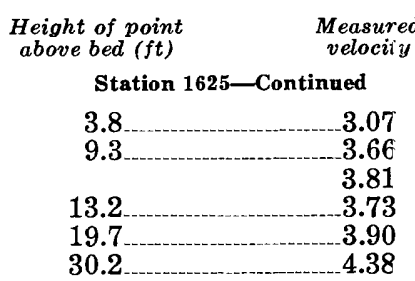

Station 1700

Oct. 11; total depth, $37.1 \mathrm{ft}$; mean time, 1405 ; gage height, $7.25 \mathrm{ft}$

$\begin{array}{rr}1.5 & 2.01 \\ 1.62 \\ 2.5 & 1.92 \\ 4.1 & 2.16 \\ 6.7 & 2.62 \\ 11.1 & 3.69 \\ 18.6 & 3.14 \\ 31.3 & 3.38\end{array}$

Station 475

Apr, 19; total depth, $15.0 \mathrm{ft}$; mean time, 1125 ; gage height, $6.46 \mathrm{ft}$

$\begin{array}{rr}1.5 & 2.33 \\ 2.2 & 2.85 \\ & 2.91 \\ 3.1 & 3.20 \\ 4.5 & 3.27 \\ & 3.20 \\ 6.6 & 3.42 \\ 9.4 & 3.63 \\ 13.5 & 3.71\end{array}$

Station 825

Apr. 18; total depth, $16.3 \mathrm{ft}$; mean time, 0905; gage height, $6.89 \mathrm{ft}$

1.5
2.2
3.2
4.7
6.9

Apr. 18 ; mean time, 0920 ; gage height, 6.89 ft

$\begin{array}{rr}1.5 & 2.73 \\ 2.2 & 3.27 \\ 3.2 & 2.85 \\ 4.7 & 2.91 \\ 6.9 & 3.63 \\ 10.1 & \end{array}$

Apr. 18 ; mean time, 0935 ; gage height, 6.88

1.5
2.2
3.2
4.7
6.9
10.1
14.5




\section{J22 CONTRIBUTIONS TO THE HYDROLOGY OF THE UNITED STATES}

TABLE 3.-Measured velocity, in feet per second, at selected points above riverbed, April 1961, April and October 1962, and April 1963-Continued

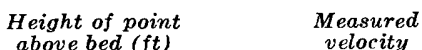

Station 825-Continued

Apr. 18 ; mean time, 1200 ; gage height, 6.89 ft

1.5
2.2
3.2
4.7
6.9
Apr. 18; mean time, $\underset{\mathrm{ft}}{1215}$; gage height, 6.89

$\begin{array}{rr}1.5 & 2.53 \\ 2.2 & 2.79 \\ 3.2 & 2.98 \\ 4.7 & 3.12 \\ 6.9 & 3.12 \\ 10.1 & 3.96 \\ 14.5 & 4.15\end{array}$

Apr. 18 ; mean time, 1230 ; gage height, 6.90 ft

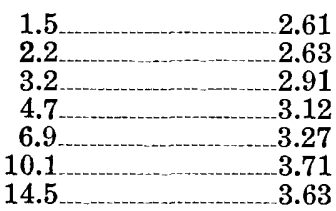

Station 1100

Apr. 17 ; total depth, $21.8 \mathrm{ft}$

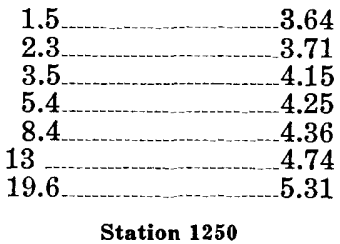

Apr. 19 ; total depth, $28.5 \mathrm{ft}$; mean time, 0935 ; gage height, $6.58 \mathrm{ft}$

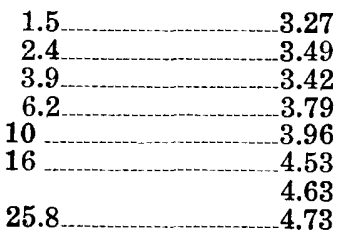
Apr. 19 ; mean time, $\underset{\mathrm{ft}}{0950 \text {; gage height, } 6.59}$

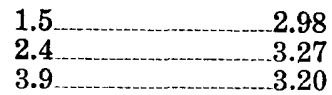

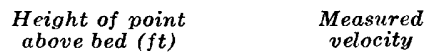

Station 1250-Continued

$\begin{array}{rr}6.2 & 3.56 \\ & 3.87 \\ 10 & 3.87 \\ & 4.25 \\ 16 & 4.53 \\ & 4.83 \\ 25.8 & 4.83\end{array}$

Apr. 19 ; mean time, 1005 ; gage height, 6.60 $\mathrm{ft}$

$\begin{array}{rr}1.5 & 3.05 \\ 2.4 & 3.27 \\ 3.9 & 3.49 \\ & 5.05 \\ 6.2 & 3.49 \\ 10 & 3.96 \\ 16 & 4.63 \\ 25.8 & 4.83\end{array}$

Apr. 19 ; mean time, 1235 ; gage height, 6.64 ft

$\begin{array}{rr}1.5 & 2.91 \\ 2.4 & 3.20 \\ 3.9 & 2.67 \\ & 2.73 \\ 6.2 & 3.56 \\ & 3.96 \\ 10 & 3.79 \\ & 3.49 \\ 16 & 4.63 \\ 25.8 & 5.06\end{array}$

Apr. 19 ; mean time, 1250 ; gage height, 6.64 ft

$\begin{array}{rr}1.5 & 2.67 \\ 2.4 & 2.85 \\ 3.9 & 2.98 \\ 6.2 & 3.98 \\ 10 & 3.83 \\ & 4.25 \\ 16 & 4.44 \\ & 4.83\end{array}$

Apr. 19 ; mean time, 1305 ; gage height, 6.64 ft

$\begin{array}{rr}1.5 & 2.61 \\ 2.4 & 2.61 \\ 3.9 & 3.36 \\ & 3.49 \\ 6.2 & 3.42 \\ 10 & 3.71 \\ 16 & 4.44 \\ & 4.63 \\ 25.8 & 4.83\end{array}$


TABLE 3.-Measured velocity, in feet per second, at selected points above riverbed, April 1961, April and October 1962, and April 1963-Continued

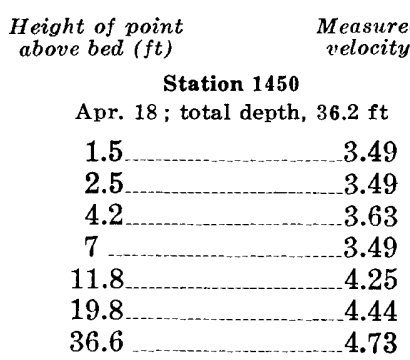

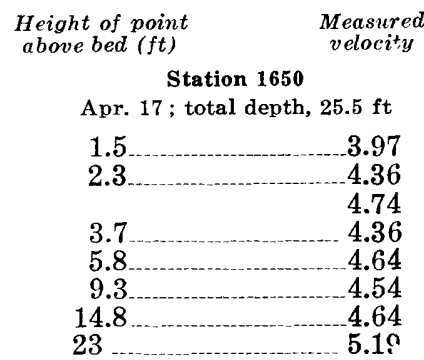

\section{COMPUTATION OF AVERAGE TURBULENCE CONSTAI'T}

The turbulence, or Karman, constant, $k$, can be computed from the equation

where

$$
k=\frac{2.30 u_{*}}{\left(V_{10 y}-V_{y}\right)},
$$

$u_{*}$ is the shear velocity equal to $\sqrt{g \overline{D S_{e}}}$, where

$g$ is the acceleration of gravity,

$D$ is the depth, and

$S_{e}$ is the energy gradient,

$V$ is the velocity, and

$y$ is the distance above the river bed.

The velocities at distances $10 y$ and $y$ above the riverbed are taken from the line of best fit of the vertical-velocity profiles. The average turbulence constant for a cross section can be obtained in two ways: it can be computed from an average $u_{*}$ and the arerage velocity differences, or it can be computed for each vertical and the individual $k$ 's averaged. According to Colby (in Jordan, 1؟35, p. 54), the turbulence constant can also be computed as

$$
k=\frac{1.39 u_{*}}{\left(V_{4 y}-V_{y}\right)}
$$

where $u_{*}$ is computed from the mean of depths at the measuring verticals and the velocity differences at distances $4 y$ and $y$ from the bed are from the 0.2 and 0.8 depth velocities obtained for the streamflow measurement. The average $u_{*}$ and average velocity differences are used in computing $k$ from the streamflow me'surement data; therefore, the averaging procedure corresponds to the first method described above. Depths and velocity differences for verticals near the banks, where bank resistance has an ap- 
preciable effect on the vertical-velocity profile, are not included in the computations of average constants.

Turbulence constants computed from data obtained on consecutive days (table 4) indicate that data from either streamflowmeasurement notes or from vertical-velocity profiles are satisfactory for computing $k$ for flows at St. Louis; Colby (in Jordan, 1965, p. 42-56) also found that the turbulence constants could be computed from either type of data. All turbulence constants for the set of data obtained April 16-20, 1963, were computed from streamflow-measurement notes. The averages of all $k$ 's computed from streamflow-measurement notes and from average velocity difference and average depth at each vertical are 0.34 and 0.35 , respectively. Colby (in Jordan, 1965, p. 54) found the averages of $k$ 's computed by the same procedures to be 0.33 and 0.35 , respectively. The average $(0.38)$ of all $k$ 's in the last column of table 4 is somewhat higher than the average from either of the other computations because of the difference in the procedures used in computing the individual $k$ 's.

In the computation of shear velocity $\left(u_{*}\right)$, the energy gradient, $S_{e}$, appears under the square-root sign. If a 5 percent error in $k$ due to incorrect energy gradient is acceptable, then the energy gradient may differ as much as \pm 10 percent from the true energy gradient. As energy gradients are likely to range from 0.3 to 0.8

TABLE 4.-Summary of turbulence constants

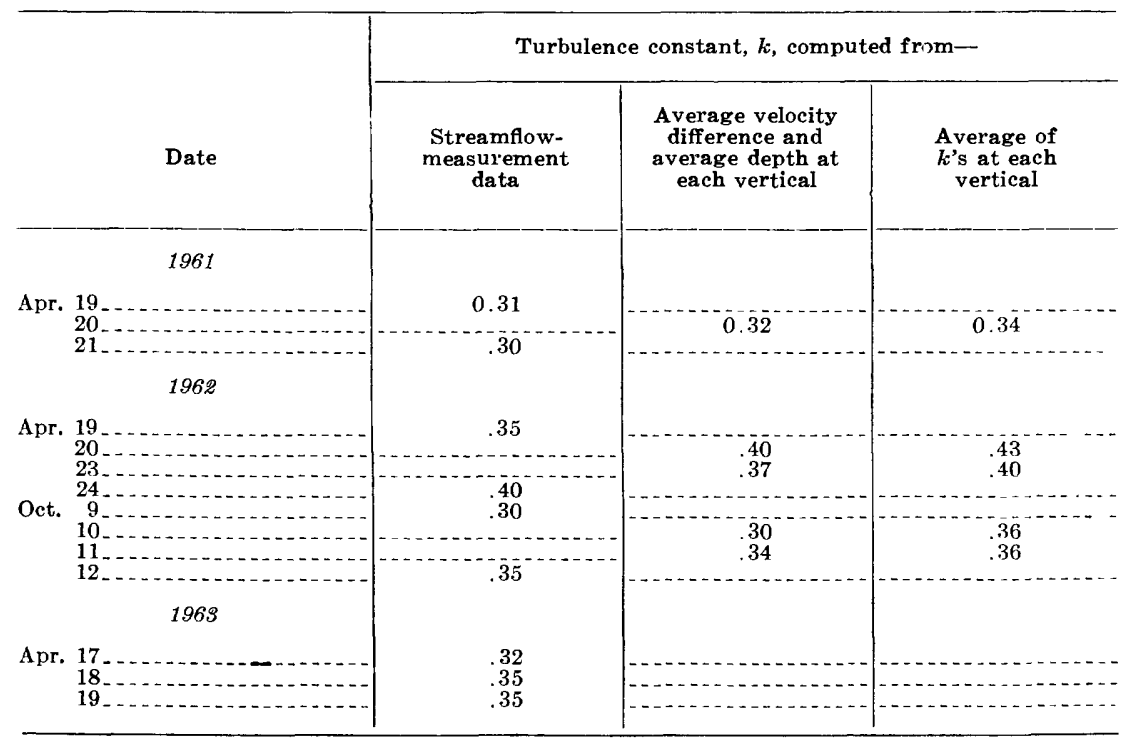


foot per mile for most flows at St. Louis, the assumed energy gradient could differ from the true energy gradient by 0.03 foot per mile for the least steep slope to 0.08 foot per mile for the steepest slope; the difference for 4 miles could range from 0.12 to 0.32 foot.

The $k$ 's were computed from the energy gradients determined for the reach from mile 181.0 to mile 177.1 or from mile 181.0 to mile 176.8; the break that generally occurs in the energy gradient in the vicinity of mile 178.9 was ignored. Although the errors caused by inaccurate measurement of the water-surface slopes over the entire reach probably are well within the 5 percent assumed as a reasonable limit, the break in the energy gradient that generally occurs at mile 178.9 could cause appreciable differences in the computed $k$ if the energy gradient upstream or downstream from mile 178.9 is used in the computation of $u_{*}$ rather than the gradient for the reach from mile 181.0 to 176.8 or 177.1. In April 1962, the slopes (table 5) upstream and downstream from mile 178.9 were within \pm 10 percent of the slope over the entire reach, but in April 1961, October 1962, and April 1963, the highest and lowest slopes differed more than 10 percent from the slope over the entire reach. The maximum difference between the computed energy gradient over the entire reach and the computed energy gradient upstream or downstream from mile 178.9 occurred in the April 1961 data; it was about 17 percent.

\section{SEDIMENT DATA}

Each set of sediment data consists of point samples, crosssection samples, bed-material samples, and water temperatures. The size distribution of material 2.0 millimeters and larger was determined by sieving, and that of material smaller than $2.0 \mathrm{~mm}$ by fall-diameter methods. The visual-accumulation-tube mothod was used for material between $1.0 \mathrm{~mm}$ and $0.062 \mathrm{~mm}$, and the pipet method for material less than $0.062 \mathrm{~mm}$.

\section{VERTICAL DISTRIBUTION OF SUSPENDED SEDIMENT}

Point-integrated samples were obtained on the $2 \mathrm{~d}, 3 \mathrm{~d}$, and 4 th days of each data-collection period except in the April 1962 period when they were obtained on 4 days. Each vertical was sampled at three to six depths, and the depths at which the samples were taken were selected so that the differences in the logarithms of $(D-y) / y$ (where $D$ is depth and $y$ is distance above the bed) were about equal. In April 1961, April 1962, and April 196?, the samples were obtained with a U.S. P-46 sampler having an addi- 
J26 CONTRIBUTIONS TO THE HYDROLOGY OF THE UNITED STATES

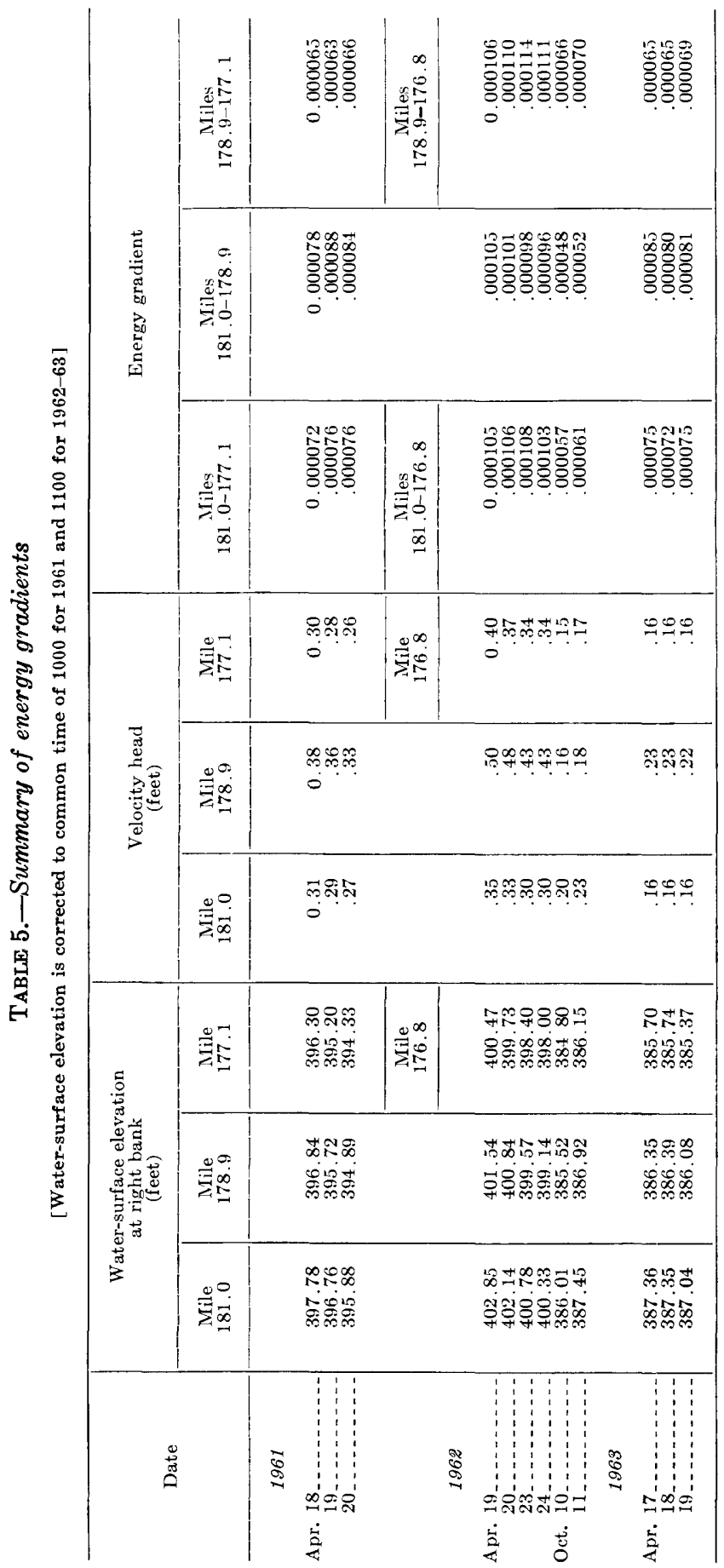


tional sounding weight suspended below. The lowest sampling point was about 1.8 feet above the bed because the additional weight prevented sampling closer. In October 1962, no sounding weight was used and the lowest sampling point was 1.5 feet above the bed. The number of verticals that could be sampled in a day depended on the concentration of sand. If the concentration of sand was relatively high, three verticals could be sampled per day; however, if the concentration was relatively low, only one or two could be sampled because a larger sample had to be obtained at each point to insure that enough material was available for a reliable analysis of particle-size distribution. The verticals at which point samples were obtained were selected to reprosent subsections of about equal flow.

The sample from each point in the vertical was analyzed for concentration of suspended sediment and particle-size distribution (table 6). The concentration for various size ranges was computed as the product of the sample concentration and the decimal portion of the sample in the size range, as determined from the size analysis. The slope, called $z_{1}$, of the relation of the logarithm of the concentration of various size ranges $\left(C_{y}\right)$ plotted against the logarithm of $(D-y) / y$ defines the vertical distribution of sediment concentration for the various size ranges of sand (pl. 2). The vertical distribution of sediment for various size ranges can be computed from the theoretical equation

where

$$
z=\frac{V_{s}}{k u_{*}}
$$

$V_{s}$ is the fall velocity of the geometric mean size of tho size range in question,

$k \quad$ is the turbulence, or Karman, constant, and

$u_{*}$ is the shear velocity equal to $\sqrt{g D S_{e}}$.

The energy gradient enters the computation of $u_{*}$ to the one-half power; therefore, the computed $z$ varies inversely with the one-half power of the energy gradient. The energy gradient upstream and downstream from mile 178.9 can vary considerably from the average gradient over the entire reach, but the percentage variation in the computed $z$ 's will be less than the percentage variation of the gradient upstream and downstream from the average gradient. The fall velocity enters to the first power in the computation of $z$, but the relation of the measured $z_{1}$ to fall velocity indicated that the power of the fall velocity should be less than one (pl. 3). The fall velocities are from a report of the U.S. Inter-Agency Committee on Water Resources (1957, table 2). The 


\section{J28 CONTRIBUTIONS TO THE HYDROLOGY OF THE UNITED STATES}

TABLE 6.-Size distribution of suspended sediment and velocity at selected points above riverbed, April 1961, April and October 1962, and April 1963

[Velocity computed from sample volume, filling time, and nozzle size]

\begin{tabular}{|c|c|c|c|c|c|c|}
\hline \multirow{3}{*}{$\underset{\text { time }}{\text { Mean }}$} & \multirow{3}{*}{$\begin{array}{l}\text { Gage } \\
\text { height } \\
\text { (feet) }\end{array}$} & \multirow{3}{*}{$\begin{array}{c}\text { Water } \\
\text { temperature } \\
\left({ }^{\circ} \mathrm{F}\right)\end{array}$} & \multirow{3}{*}{$\begin{array}{c}\text { Height } \\
\text { of point } \\
\text { above bed } \\
\text { (feet) }\end{array}$} & \multirow{3}{*}{$\begin{array}{l}\text { Velocity } \\
\text { (fps) }\end{array}$} & \multicolumn{2}{|r|}{ Suspended sediment } \\
\hline & & & & & Concen- & $\begin{array}{l}\text { Percent finer than indirated } \\
\operatorname{siz}^{\circ}(\mathrm{mm})\end{array}$ \\
\hline & & & & & $(\mathrm{ppm})$ & $0.062|0.125| \mathrm{C} .250|0.500| 1.000$ \\
\hline
\end{tabular}

1961

Station 465

Apr. 20; total depth, $25.8 \mathrm{ft}$

\begin{tabular}{|c|c|c|c|c|c|c|c|c|c|c|}
\hline $\begin{array}{l}1357 \\
1424 \\
1452 \\
1603\end{array}$ & 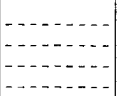 & $\begin{array}{l}52 \\
52 \\
52 \\
52\end{array}$ & $\begin{array}{r}1.8 \\
6.4 \\
14.2 \\
21.9\end{array}$ & $\begin{array}{l}2.49 \\
3.37 \\
4.16 \\
4.57\end{array}$ & $\begin{array}{r}1,200 \\
1,150 \\
1,050 \\
987\end{array}$ & $\begin{array}{l}81 \\
85 \\
90 \\
94\end{array}$ & $\begin{array}{l}87 \\
91 \\
96 \\
98\end{array}$ & $\begin{array}{r}99 \\
100 \\
100 \\
100\end{array}$ & \begin{tabular}{c}
100 \\
\hdashline.- \\
\hdashline.-- \\
$-\ldots$
\end{tabular} & 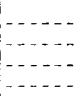 \\
\hline
\end{tabular}

Station 800

Apr. 19; total depth, $31.8 \mathrm{ft}$

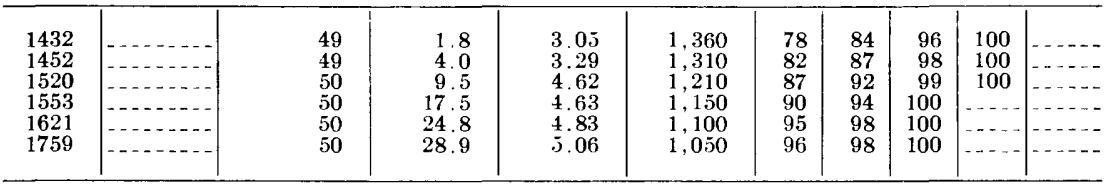

Station 1050

Apr. 20; total depth, $36.2 \mathrm{ft}$

\begin{tabular}{|c|c|c|c|c|c|c|c|c|c|c|}
\hline $\begin{array}{l}1102 \\
1118 \\
1132 \\
1152 \\
1216 \\
1250\end{array}$ & 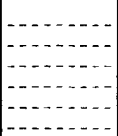 & $\begin{array}{l}50 \\
50 \\
50 \\
50 \\
50 \\
50\end{array}$ & $\begin{array}{r}1.8 \\
4.7 \\
10.9 \\
19.9 \\
28.2 \\
32.9\end{array}$ & $\begin{array}{l}1.59 \\
3.45 \\
4.16 \\
4.21 \\
5.08 \\
5.21\end{array}$ & $\begin{array}{r}1,070 \\
986 \\
902 \\
876 \\
788 \\
766\end{array}$ & $\begin{array}{l}74 \\
79 \\
85 \\
88 \\
93 \\
94\end{array}$ & $\begin{array}{l}81 \\
86 \\
92 \\
94 \\
97 \\
98\end{array}$ & $\begin{array}{r}92 \\
97 \\
100 \\
100 \\
100 \\
100\end{array}$ & $\begin{array}{c}100 \\
100 \\
\cdots \\
\cdots\end{array}$ & 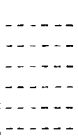 \\
\hline
\end{tabular}

Station 1250

Apr. 18; total depth, $41.2 \mathrm{ft}$

\begin{tabular}{|c|c|c|c|c|c|c|c|c|c|c|}
\hline $\begin{array}{l}1532 \\
1550 \\
1604\end{array}$ & 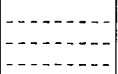 & $\begin{array}{l}48 \\
48 \\
48\end{array}$ & $\begin{array}{r}1.8 \\
4.5 \\
11.1\end{array}$ & $\begin{array}{l}1.99 \\
4.64 \\
4.30\end{array}$ & $\begin{array}{l}1,560 \\
1,170 \\
1,120\end{array}$ & $\begin{array}{l}61 \\
80 \\
83\end{array}$ & $\begin{array}{l}66 \\
86 \\
88\end{array}$ & $\begin{array}{l}79 \\
98 \\
99\end{array}$ & $\begin{array}{r}98 \\
100 \\
100\end{array}$ & $\begin{array}{c}100 \\
-\ldots\end{array}$ \\
\hline
\end{tabular}

Station 1250

Apr. 20; total depth, $39.5 \mathrm{ft}$

\begin{tabular}{|c|c|c|c|c|c|c|c|c|c|c|}
\hline $\begin{array}{l}0840 \\
0856 \\
0911 \\
0933 \\
0954 \\
1018\end{array}$ & 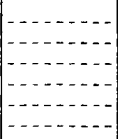 & $\begin{array}{l}49 \\
49 \\
49 \\
49 \\
49 \\
49\end{array}$ & $\begin{array}{r}1.8 \\
5.2 \\
11.9 \\
21.7 \\
30.8 \\
35.9\end{array}$ & $\begin{array}{l}3.99 \\
4.44 \\
5.41 \\
5.21 \\
5.34 \\
5.47\end{array}$ & $\begin{array}{r}1,000 \\
808 \\
711 \\
640 \\
634 \\
578\end{array}$ & $\begin{array}{l}59 \\
70 \\
81 \\
84 \\
87 \\
90\end{array}$ & $\begin{array}{l}67 \\
78 \\
89 \\
90 \\
94 \\
96\end{array}$ & $\begin{array}{r}83 \\
96 \\
100 \\
100 \\
100 \\
100\end{array}$ & \begin{tabular}{c}
100 \\
100 \\
$-\cdots$ \\
\hdashline-1 \\
\hdashline- \\
-
\end{tabular} & $\mid$\begin{tabular}{l}
$-\cdots$ \\
$-\cdots$ \\
\hdashline$-\cdots$ \\
$-\cdots$ \\
$-\cdots$ \\
$-\cdots$ \\
-
\end{tabular} \\
\hline
\end{tabular}

Station 1440

Apr. 19; total depth, $48.2 \mathrm{ft}$

\begin{tabular}{|c|c|c|c|c|c|c|c|c|c|c|}
\hline $\begin{array}{l}1012 \\
1034 \\
1052 \\
1117 \\
1145 \\
1210\end{array}$ & 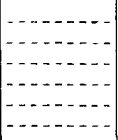 & $\begin{array}{l}48 \\
48 \\
48 \\
46 \\
46 \\
46\end{array}$ & $\begin{array}{r}1.8 \\
5.3 \\
13.0 \\
25.4 \\
37.1 \\
43.8\end{array}$ & $\begin{array}{l}2.87 \\
3.53 \\
4.28 \\
5.74 \\
6.17 \\
5.89\end{array}$ & $\begin{array}{r}862 \\
1,060 \\
720 \\
547 \\
420 \\
418\end{array}$ & $\begin{array}{l}56 \\
52 \\
69 \\
80 \\
89 \\
92\end{array}$ & $\begin{array}{l}61 \\
59 \\
77 \\
88 \\
93 \\
96\end{array}$ & $\begin{array}{r}99 \\
93 \\
99 \\
100 \\
100 \\
100\end{array}$ & $\begin{array}{c}100 \\
100 \\
100 \\
\cdots \\
\cdots \\
\cdots\end{array}$ & 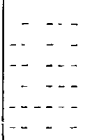 \\
\hline
\end{tabular}


TABLE 6.-Size distribution of suspended sediment and velocity at selected points above riverbed, April 1961, April and October 1962, and April 1963Continued

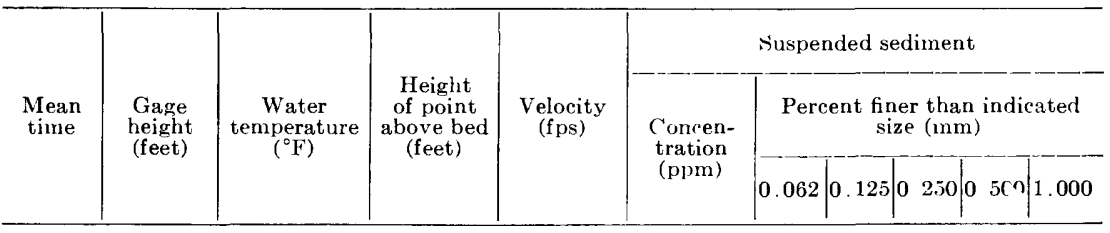

Station 1580

Apr. 18; total depth, $41.8 \mathrm{ft}$

\begin{tabular}{|c|c|c|c|c|c|c|c|c|c|c|}
\hline $\begin{array}{l}1111 \\
1136 \\
1155 \\
1222 \\
1255 \\
1325\end{array}$ & $\mid \begin{array}{c}\cdots \\
\cdots \\
-\cdots\end{array}$ & $\begin{array}{l}45 \\
45 \\
45 \\
45 \\
46 \\
46\end{array}$ & $\begin{array}{r}1.8 \\
4.6 \\
11.3 \\
22.2 \\
32.2 \\
38.0\end{array}$ & $\begin{array}{l}2.61 \\
2.56 \\
4.05 \\
4.59 \\
4.96 \\
5.21\end{array}$ & $\begin{array}{r}1,210 \\
956 \\
655 \\
462 \\
350 \\
312\end{array}$ & $\begin{array}{l}33 \\
40 \\
60 \\
70 \\
87 \\
93\end{array}$ & $\begin{array}{l}38 \\
47 \\
66 \\
77 \\
92 \\
96\end{array}$ & $\begin{array}{r}99 \\
98 \\
99 \\
100 \\
100 \\
100\end{array}$ & \begin{tabular}{c}
$10 \mathrm{C}$ \\
$10 \mathrm{C}$ \\
$10 \mathrm{C}$ \\
$\cdots \ldots$ \\
\hdashline$\ldots$ \\
\hdashline$\ldots$
\end{tabular} & 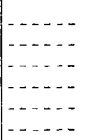 \\
\hline
\end{tabular}

\section{2}

Station 400

Apr. 23; total depth, $33.3 \mathrm{ft}$

\begin{tabular}{|c|c|c|c|c|c|c|c|c|c|c|}
\hline $\begin{array}{l}1340 \\
1357 \\
1428 \\
1440 \\
1501\end{array}$ & 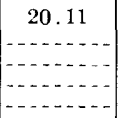 & $\begin{array}{c}52^{2} \\
55^{2}\end{array}$ & $\begin{array}{r}1.8 \\
5.7 \\
14.0 \\
23.3 \\
30.3\end{array}$ & $\begin{array}{l}2.52 \\
3.69 \\
4.46 \\
4.46 \\
5.48\end{array}$ & $\begin{array}{r}2,000 \\
789 \\
603 \\
478 \\
406\end{array}$ & $\begin{array}{l}19 \\
47 \\
63 \\
73 \\
86\end{array}$ & $\begin{array}{l}24 \\
57 \\
71 \\
84 \\
92\end{array}$ & $\begin{array}{r}51 \\
76 \\
88 \\
96 \\
100\end{array}$ & $\begin{array}{r}97 \\
98 \\
10 C \\
100\end{array}$ & $\begin{array}{c}100 \\
100 \\
\ldots \ldots \\
\ldots \ldots\end{array}$ \\
\hline
\end{tabular}

Station 725

Apr. 19; total depth, $38.7 \mathrm{ft}$

\begin{tabular}{|c|c|c|c|c|c|c|c|c|c|c|}
\hline $\begin{array}{l}1454 \\
1516 \\
1530 \\
1545 \\
1600\end{array}$ & \begin{tabular}{c}
22.06 \\
$-\ldots \ldots$ \\
\hdashline$\ldots \ldots$ \\
\hdashline$\ldots \ldots$ \\
\hdashline$\ldots \ldots$
\end{tabular} & \begin{tabular}{r}
51 \\
\hdashline$\ldots 1$ \\
51
\end{tabular} \mid & $\begin{array}{r}1.8 \\
6.4 \\
16.2 \\
28.3 \\
35.2\end{array}$ & $\begin{array}{l}4.25 \\
3.70 \\
3.96 \\
6.12 \\
6.57\end{array}$ & $\begin{array}{l}964 \\
734 \\
553 \\
452 \\
390\end{array}$ & $\begin{array}{l}36 \\
49 \\
62 \\
72 \\
81\end{array}$ & $\begin{array}{l}42 \\
59 \\
75 \\
84 \\
91\end{array}$ & $\begin{array}{l}52 \\
72 \\
88 \\
96 \\
99\end{array}$ & $\begin{array}{r}87 \\
96 \\
98 \\
98 \\
100\end{array}$ & $\begin{array}{r}99 \\
100 \\
100 \\
100 \\
-\end{array}$ \\
\hline
\end{tabular}

Station 725

Apr. 24; total depth, $35.3 \mathrm{ft}$

\begin{tabular}{|c|c|c|c|c|c|c|c|c|c|c|}
\hline $\begin{array}{l}1438 \\
1454 \\
1508 \\
1538 \\
1615\end{array}$ & \begin{tabular}{c}
19.64 \\
\hdashline-1 \\
-1
\end{tabular} & $\begin{array}{l}57 \\
55 \\
55 \\
55 \\
55\end{array}$ & $\begin{array}{r}1.8 \\
6.0 \\
14.8 \\
26.0 \\
32.1\end{array}$ & $\begin{array}{l}3.30 \\
4.66 \\
5.24 \\
5.74 \\
6.75\end{array}$ & $\begin{array}{l}541 \\
540 \\
536 \\
307 \\
319\end{array}$ & $\begin{array}{l}51 \\
55 \\
70 \\
78 \\
84\end{array}$ & $\begin{array}{l}64 \\
67 \\
82 \\
89 \\
92\end{array}$ & $\begin{array}{l}83 \\
86 \\
96 \\
99 \\
99\end{array}$ & $\begin{array}{r}98 \\
100 \\
100 \\
100 \\
100\end{array}$ & \begin{tabular}{c}
100 \\
$\ldots \ldots$ \\
\hdashline$\ldots$ \\
\hdashline$\ldots$
\end{tabular} \\
\hline
\end{tabular}

Station 985

Apr. 20; total depth, $41.6 \mathrm{ft}$

\begin{tabular}{|c|c|c|c|c|c|c|c|c|c|c|}
\hline $\begin{array}{l}1356 \\
1414 \\
1432 \\
1452 \\
1718\end{array}$ & $\mid$\begin{tabular}{c}
21.34 \\
\hdashline$-\ldots \ldots$ \\
\hdashline$-\ldots$. \\
\hdashline$-\ldots$. \\
\hdashline$\ldots$
\end{tabular} & $\begin{array}{r}50 \\
50 \\
50 \\
50 \\
\ldots\end{array}$ & $\begin{array}{r}1.8 \\
5.8 \\
16.2 \\
30.0 \\
37.9\end{array}$ & $\begin{array}{l}3.00 \\
3.83 \\
4.38 \\
5.46 \\
5.76\end{array}$ & $\begin{array}{l}844 \\
520 \\
436 \\
333 \\
317\end{array}$ & $\begin{array}{l}34 \\
50 \\
63 \\
75 \\
80\end{array}$ & $\begin{array}{l}42 \\
65 \\
75 \\
87 \\
89\end{array}$ & $\begin{array}{l}58 \\
82 \\
90 \\
98 \\
99\end{array}$ & $\begin{array}{r}95 \\
99 \\
100 \\
100 \\
100\end{array}$ & $\begin{array}{l}100 \\
100 \\
\ldots \ldots\end{array}$ \\
\hline
\end{tabular}

Station 985

A pr. 23; total depth, $36.3 \mathrm{ft}$

\begin{tabular}{|c|c|c|c|c|c|c|c|c|c|c|}
\hline $\begin{array}{l}1616 \\
1633 \\
1713 \\
1756 \\
1818\end{array}$ & 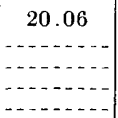 & \begin{tabular}{c}
55 \\
-56 \\
\hdashline-10
\end{tabular} & $\begin{array}{r}1.8 \\
6.2 \\
15.2 \\
26.4 \\
33.0\end{array}$ & $\begin{array}{l}4.14 \\
4.55 \\
4.91 \\
5.20 \\
5.44\end{array}$ & $\begin{array}{l}447 \\
421 \\
364 \\
322 \\
270\end{array}$ & $\begin{array}{l}44 \\
56 \\
64 \\
71 \\
84\end{array}$ & $\begin{array}{l}55 \\
67 \\
78 \\
84 \\
91\end{array}$ & $\begin{array}{l}87 \\
84 \\
92 \\
97 \\
99\end{array}$ & $\begin{array}{l}100 \\
100 \\
100 \\
100 \\
100\end{array}$ & $\begin{array}{c}\cdots \\
\cdots \\
\cdots \\
\cdots \\
\cdots \\
\cdots\end{array}$ \\
\hline
\end{tabular}




\section{J30 CONTRIBUTIONS TO THE HYDROLOGY OF THE UNITED STATES}

TABLE 6.-Size distribution of suspended sediment and velocity at selected points above riverbed, April 1961, April and October 1962, and April 1963Continued

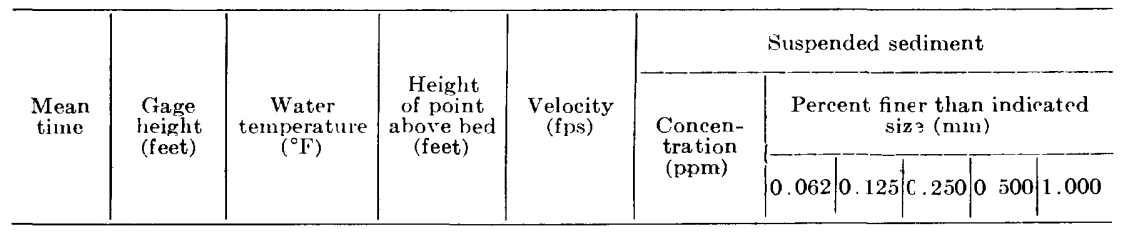

Station 1220

A pr. 19; total depth, $43.2 \mathrm{ft}$

\begin{tabular}{|c|c|c|c|c|c|c|c|c|c|c|}
\hline $\begin{array}{l}1632 \\
1646 \\
1702 \\
1717 \\
1743\end{array}$ & $\mid$\begin{tabular}{c}
22.00 \\
- \\
\hdashline-1. \\
\hdashline
\end{tabular} & $\begin{array}{l}50 \\
50 \\
50 \\
50 \\
50\end{array}$ & $\begin{array}{r}1.8 \\
6.0 \\
16.9 \\
31.1 \\
39.3\end{array}$ & $\begin{array}{l}3.42 \\
3.94 \\
5.94 \\
7.02 \\
6.99\end{array}$ & $\begin{array}{l}648 \\
518 \\
291 \\
241 \\
225\end{array}$ & $\begin{array}{l}34 \\
41 \\
73 \\
84 \\
87\end{array}$ & $\begin{array}{l}40 \\
48 \\
80 \\
87 \\
92\end{array}$ & $\begin{array}{l}50 \\
61 \\
93 \\
97 \\
98\end{array}$ & $\begin{array}{l}100 \\
100 \\
100 \\
100 \\
100\end{array}$ & 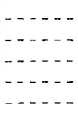 \\
\hline
\end{tabular}

Station 1220

A pr. 24; total depth, $41.6 \mathrm{ft}$

\begin{tabular}{|c|c|c|c|c|c|c|c|c|c|c|}
\hline $\begin{array}{l}1210 \\
1229 \\
1248 \\
1308 \\
1334\end{array}$ & $\mid$\begin{tabular}{c}
19.67 \\
\hdashline-1 \\
\hdashline
\end{tabular} & $\begin{array}{l}54 \\
54 \\
54 \\
54 \\
54\end{array}$ & $\begin{array}{r}1.8 \\
5.8 \\
16.2 \\
30.0 \\
37.9\end{array}$ & $\begin{array}{l}3.84 \\
4.15 \\
4.95 \\
6.02 \\
6.46\end{array}$ & $\begin{array}{l}422 \\
368 \\
282 \\
235 \\
219\end{array}$ & $\begin{array}{l}48 \\
57 \\
72 \\
85 \\
90\end{array}$ & $\begin{array}{l}55 \\
64 \\
80 \\
91 \\
95\end{array}$ & $\begin{array}{r}67 \\
75 \\
91 \\
98 \\
100\end{array}$ & $\begin{array}{r}99 \\
98 \\
100 \\
100 \\
- \\
-\end{array}$ & $\begin{array}{c}100 \\
100 \\
\ldots \ldots \\
\ldots \ldots\end{array}$ \\
\hline
\end{tabular}

Station 1450

A pr. 20; total depth, $53.6 \mathrm{ft}$

\begin{tabular}{|c|c|c|c|c|c|c|c|c|c|c|}
\hline $\begin{array}{l}1020 \\
1036 \\
1056 \\
1126 \\
1154\end{array}$ & $\mid$\begin{tabular}{c}
21.45 \\
\hdashline
\end{tabular} & $\begin{array}{l}50 \\
50 \\
50 \\
50 \\
50\end{array}$ & $\begin{array}{r}1.8 \\
6.4 \\
19.8 \\
38.1 \\
48.8\end{array}$ & $\begin{array}{l}3.55 \\
4.63 \\
5.24 \\
6.50 \\
6.71\end{array}$ & $\begin{array}{r}1,0: 20 \\
4.58 \\
320 \\
237 \\
214\end{array}$ & $\begin{array}{l}19 \\
41 \\
57 \\
77 \\
86\end{array}$ & $\begin{array}{l}20 \\
44 \\
62 \\
82 \\
90\end{array}$ & $\begin{array}{l}32 \\
58 \\
74 \\
90 \\
96\end{array}$ & $\begin{array}{r}100 \\
98 \\
100 \\
100 \\
100\end{array}$ & $\begin{array}{c}100 \\
-\end{array}$ \\
\hline
\end{tabular}

Station 1450

Apr. 23; total depth, $49.8 \mathrm{ft}$

\begin{tabular}{|c|c|c|c|c|c|c|c|c|c|c|}
\hline $\begin{array}{l}1028 \\
1118 \\
1136 \\
1222 \\
1245\end{array}$ & \begin{tabular}{c}
20.14 \\
-- \\
\hdashline-
\end{tabular} & $\begin{array}{l}53 \\
53 \\
53 \\
53 \\
53\end{array}$ & $\begin{array}{r}1.8 \\
7.0 \\
19.4 \\
35.9 \\
45.3\end{array}$ & $\begin{array}{l}4.17 \\
4.60 \\
5.02 \\
5.24 \\
5.86\end{array}$ & $\begin{array}{l}561 \\
343 \\
262 \\
226 \\
210\end{array}$ & $\begin{array}{l}34 \\
55 \\
73 \\
84 \\
88\end{array}$ & $\begin{array}{l}38 \\
57 \\
79 \\
88 \\
92\end{array}$ & $\begin{array}{l}48 \\
69 \\
86 \\
95 \\
97\end{array}$ & $\begin{array}{r}99 \\
100 \\
100 \\
100 \\
100\end{array}$ & $\begin{array}{c}100 \\
-\ldots \ldots \\
-\ldots \\
-\ldots \\
\ldots \ldots\end{array}$ \\
\hline
\end{tabular}

Station 1670

Apr. 19; total depth, $49.4 \mathrm{ft}$

\begin{tabular}{|c|c|c|c|c|c|c|c|c|c|c|}
\hline $\begin{array}{l}1257 \\
1312 \\
1326 \\
1346 \\
1401\end{array}$ & \begin{tabular}{c}
22.10 \\
- \\
\hdashline \\
\hdashline
\end{tabular} & $\begin{array}{l}51 \\
51 \\
51 \\
51 \\
51\end{array}$ & $\begin{array}{r}1.8 \\
6.9 \\
19.3 \\
35.6 \\
45.0\end{array}$ & $\begin{array}{l}4.75 \\
4.72 \\
5.99 \\
5.37 \\
5.77\end{array}$ & $\begin{array}{l}418 \\
283 \\
222 \\
207 \\
199\end{array}$ & $\begin{array}{l}43 \\
69 \\
83 \\
84 \\
91\end{array}$ & $\begin{array}{l}46 \\
73 \\
85 \\
87 \\
95\end{array}$ & $\begin{array}{l}75 \\
87 \\
94 \\
94 \\
98\end{array}$ & $\begin{array}{r}100 \\
100 \\
100 \\
98 \\
100\end{array}$ & 100 \\
\hline
\end{tabular}

Station 1685

Apr. 24; total depth, $45.1 \mathrm{ft}$

\begin{tabular}{|c|c|c|c|c|c|c|c|c|c|c|}
\hline $\begin{array}{l}0928 \\
0944 \\
1002 \\
1028 \\
1056\end{array}$ & \begin{tabular}{c}
19.72 \\
\hdashline$-\ldots$ \\
\hdashline-1 \\
\hdashline
\end{tabular} & $\begin{array}{l}55 \\
54 \\
54 \\
54 \\
54\end{array}$ & $\begin{array}{r}1.8 \\
6.3 \\
17.6 \\
32.5 \\
41.0\end{array}$ & $\begin{array}{l}3.72 \\
4.00 \\
4.55 \\
5.23 \\
5.35\end{array}$ & $\begin{array}{l}374 \\
235 \\
208 \\
198 \\
187\end{array}$ & $\begin{array}{l}53 \\
78 \\
90 \\
92 \\
94\end{array}$ & $\begin{array}{l}55 \\
80 \\
92 \\
94 \\
96\end{array}$ & $\begin{array}{l}95 \\
94 \\
98 \\
99 \\
99\end{array}$ & $\begin{array}{l}100 \\
100 \\
100 \\
100 \\
100\end{array}$ & $\mid \begin{array}{c}\cdots \\
\cdots \\
\cdots \\
\cdots \\
\cdots \\
\cdots\end{array}$ \\
\hline
\end{tabular}


TABLE 6.-Size distribution of suspended sediment and velocity at solected points above riverbed, April 1961, April and October 1962, and April 1963Continued

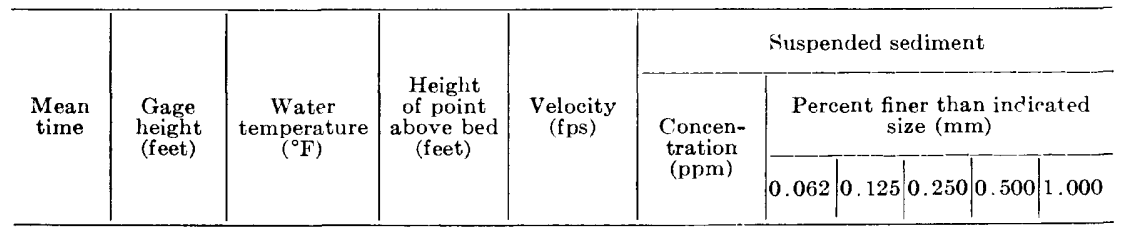

\section{Station 600}

Oct. 11; total depth, $20.3 \mathrm{ft}$

\begin{tabular}{|c|c|c|c|c|c|c|c|c|c|c|}
\hline $\begin{array}{l}0825 \\
0850 \\
0921 \\
1008 \\
1137\end{array}$ & $\begin{array}{l}7.05 \\
7.08 \\
7.11 \\
7.15 \\
7.20\end{array}$ & $\begin{array}{r}67 \\
67 \\
68\end{array}$ & $\begin{array}{r}1.5 \\
4.7 \\
10.0 \\
15.6 \\
18.3\end{array}$ & $\begin{array}{l}2.58 \\
3.24 \\
3.55 \\
3.83 \\
3.94\end{array}$ & $\begin{array}{l}513 \\
450 \\
430 \\
418 \\
403\end{array}$ & $\begin{array}{l}86 \\
95 \\
97 \\
98 \\
98\end{array}$ & $\begin{array}{r}90 \\
98 \\
98 \\
99 \\
100\end{array}$ & $\begin{array}{r}99 \\
100 \\
100 \\
100 \\
-\end{array}$ & \begin{tabular}{c}
100 \\
$\cdots-$ \\
\hdashline- \\
\hdashline- \\
$\cdots$
\end{tabular} & $\mid \begin{array}{c}\ldots \ldots \\
\cdots-\ldots \\
\cdots \\
\cdots \\
\cdots \\
\cdots\end{array}$ \\
\hline
\end{tabular}

Station 1160

Oct. 10; total depth, $26.9 \mathrm{ft}$

\begin{tabular}{|c|c|c|c|c|c|c|c|c|c|c|}
\hline $\begin{array}{l}0902 \\
0928 \\
1004 \\
1117 \\
1241\end{array}$ & $\begin{array}{l}5.81 \\
5.81 \\
5.78 \\
5.76 \\
5.76\end{array}$ & \begin{tabular}{c}
66 \\
$\ldots$ \\
\hdashline 6 \\
6
\end{tabular} & $\begin{array}{r}1.5 \\
5.2 \\
12.3 \\
21.0 \\
24.8\end{array}$ & $\begin{array}{l}2.65 \\
3.46 \\
3.70 \\
4.07 \\
4.11\end{array}$ & $\begin{array}{l}497 \\
323 \\
283 \\
264 \\
252\end{array}$ & $\begin{array}{l}53 \\
80 \\
88 \\
93 \\
96\end{array}$ & $\begin{array}{l}65 \\
87 \\
94 \\
96 \\
99\end{array}$ & $\begin{array}{r}96 \\
100 \\
100 \\
100 \\
100\end{array}$ & \begin{tabular}{c}
99 \\
$-\ldots-$ \\
\hdashline-2 \\
\hdashline- \\
\hdashline-
\end{tabular} & \begin{tabular}{c}
100 \\
$\ldots \ldots$ \\
$\ldots \ldots$ \\
\hdashline$\ldots \ldots$ \\
$\ldots$
\end{tabular} \\
\hline
\end{tabular}

Station 1550

Oct. 9; total depth, $29.3 \mathrm{ft}$

\begin{tabular}{|c|c|c|c|c|c|c|c|c|c|c|}
\hline $\begin{array}{l}0939 \\
1016 \\
1205 \\
1312 \\
1429\end{array}$ & $\begin{array}{l}6.14 \\
6.13 \\
6.11 \\
6.09 \\
6.07\end{array}$ & $\begin{array}{c}65 \\
677^{-}\end{array}$ & $\begin{array}{r}1.5 \\
5.4 \\
13.0 \\
22.5 \\
26.5\end{array}$ & $\begin{array}{l}2.39 \\
3.74 \\
3.92 \\
4.48 \\
4.53\end{array}$ & $\begin{array}{l}238 \\
166 \\
145 \\
122 \\
124\end{array}$ & $\begin{array}{l}48 \\
64 \\
75 \\
85 \\
86\end{array}$ & $\begin{array}{l}57 \\
75 \\
84 \\
91 \\
91\end{array}$ & $\begin{array}{r}91 \\
98 \\
100 \\
100 \\
100\end{array}$ & \begin{tabular}{r}
98 \\
100 \\
\hdashline- \\
-2
\end{tabular} & $\mid \begin{array}{c}100 \\
\ldots \ldots \\
\ldots \ldots \\
\cdots \ldots\end{array}$ \\
\hline
\end{tabular}

1963

Station 475

Apr. 19 ; total depth $15.2 \mathrm{ft}$

\begin{tabular}{|c|c|c|c|c|c|c|c|c|c|c|}
\hline $\begin{array}{l}1244 \\
1258 \\
1318 \\
1400\end{array}$ & $\begin{array}{l}6.64 \\
6.64 \\
6.65 \\
6.67\end{array}$ & $\begin{array}{l}64 \\
64 \\
64 \\
64\end{array}$ & $\begin{array}{r}1.8 \\
4.4 \\
\mathbf{8 . 2} \\
12.2\end{array}$ & $\begin{array}{l}3.11 \\
3.17 \\
3.46 \\
4.03\end{array}$ & $\begin{array}{l}593 \\
501 \\
480 \\
405\end{array}$ & $\begin{array}{l}68 \\
79 \\
86 \\
98\end{array}$ & $\begin{array}{l}73 \\
83 \\
90 \\
96\end{array}$ & $\begin{array}{r}98 \\
99 \\
100 \\
100\end{array}$ & \begin{tabular}{c}
100 \\
100 \\
\hdashline- \\
-
\end{tabular} & $\mid$\begin{tabular}{l}
$\ldots$ \\
\hdashline$-\ldots$ \\
\hdashline-
\end{tabular} \\
\hline
\end{tabular}

Station 825

Apr. 18; total depth, $16.5 \mathrm{ft}$

\begin{tabular}{l|l|l|r|r|r|r|r|r|r|r}
\hline 1225 & 6.90 & 63 & 1.8 & 2.49 & 1,380 & 52 & 57 & 96 & 100 & $\ldots \ldots$ \\
1323 & 6.90 & 63 & 11.5 & 4.01 & 358 & 83 & 86 & 100 & $\ldots \ldots$ \\
1406 & 6.90 & $\ldots \ldots \ldots$ & 14.0 & 4.18 & 327 & 89 & 93 & 100 & $\ldots \ldots$ \\
\hline
\end{tabular}

Station 1100

Apr. 17; total depth, $23.5 \mathrm{ft}$

\begin{tabular}{|c|c|c|c|c|c|c|c|c|c|c|}
\hline $\begin{array}{l}1258 \\
1323 \\
1340 \\
1402 \\
1440\end{array}$ & $\begin{array}{l}6.87 \\
6.87 \\
6.88 \\
6.89 \\
6.93\end{array}$ & \begin{tabular}{c}
61 \\
$\ldots \ldots \ldots \ldots$ \\
$\ldots \ldots-\ldots-$ \\
\hdashline$\ldots \ldots$
\end{tabular} & $\begin{array}{r}1.8 \\
5.1 \\
11.2 \\
17.5 \\
21.4\end{array}$ & $\begin{array}{l}2.80 \\
4.31 \\
4.47 \\
5.23 \\
5.54\end{array}$ & $\begin{array}{r}1,360 \\
441 \\
285 \\
256 \\
204\end{array}$ & $\begin{array}{l}16 \\
47 \\
71 \\
78 \\
88\end{array}$ & $\begin{array}{l}19 \\
55 \\
79 \\
84 \\
92\end{array}$ & $\begin{array}{r}87 \\
100 \\
100 \\
100 \\
100\end{array}$ & \begin{tabular}{c}
99 \\
$\ldots$ \\
\hdashline- \\
\hdashline- \\
\hdashline$\ldots$
\end{tabular} & $\mid \begin{array}{c}100 \\
\ldots \ldots \\
\ldots \\
\ldots\end{array}$ \\
\hline
\end{tabular}


TABLE 6.-Size distribution of suspended sediment and velocity at selected points above riverbed, April 1961, April and October 1962, and April 1963Continued

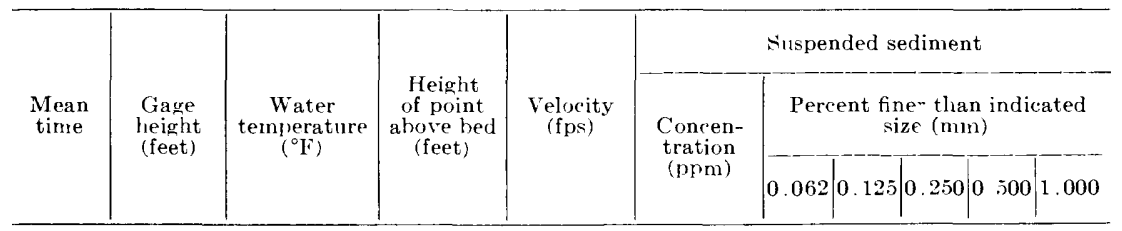

Station 1250

Apr. 19; total depth, $28.5 \mathrm{ft}$

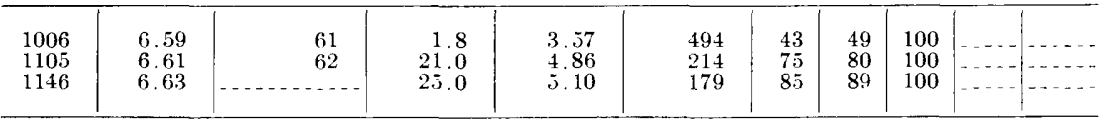

Station 1450

Apr. 18; total depth, $39.0 \mathrm{ft}$

\begin{tabular}{|c|c|c|c|c|c|c|c|c|c|c|}
\hline $\begin{array}{l}0908 \\
1010 \\
1100\end{array}$ & $\begin{array}{l}6.89 \\
6.89 \\
6.89\end{array}$ & $\begin{array}{r}61 \\
60 \\
\ldots \ldots \ldots\end{array}$ & $\begin{array}{r}1.8 \\
28.2 \\
33.5\end{array}$ & $\begin{array}{l}2.91 \\
4.66 \\
4.81\end{array}$ & $\begin{array}{r}542 \\
99 \\
92\end{array}$ & $\begin{array}{l}19 \\
82 \\
89\end{array}$ & $\begin{array}{l}22 \\
85 \\
91\end{array}$ & $\begin{array}{l}100 \\
100 \\
100\end{array}$ & $\begin{array}{c}\cdots- \\
\cdots- \\
\cdots\end{array}$ & $\mid \begin{array}{l}-\cdots \\
-\cdots \\
\cdots\end{array}$ \\
\hline
\end{tabular}

Station 1650

Apr. 17; total depth, $27.5 \mathrm{ft}$

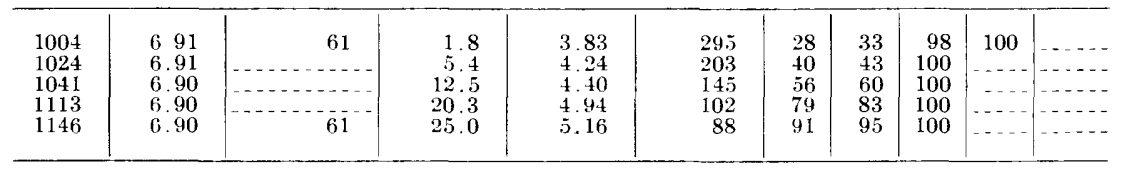

value of the power varies widely for individual vєrticals, but the average is about 0.69 for all measurements obtained for the comprehensive sets of data.

Other investigators (Anderson, 1942; Colby and Hembree, 1955) have presented data which show that $z_{1}$ 's for sand sizes vary with about the 0.7 power of the fall velocity. The relation of $z_{1}$ to fall velocity, however, seems to vary with streamflow for the deep flows at St. Louis. In April 1962, the streamflow was more than 300,000 cubic feet per second and $z_{1}$ varied, on the average, with about the 0.8 power of the fall velocity; in April 1961, the streamflow was near $250,000 \mathrm{cfs}$ and $z_{1}$ varied with about tho 0.7 power of the fall velocity. In October 1962 and April 1963, the streamflow was about $130,000 \mathrm{cfs}$ and the $z_{1}$ 's varied with about the 0.69 and 0.54 powers of the fall velocities, respectively. Streamflow and mean velocity are rather closely related for the Mississippi River at St. Louis, and the average $z_{1}$ may, thereforo, correlate with mean velocity and, possibly to some extent, with temperature; there are too few comprehensive sets of data available for a check. 


\section{CROSS-SECTION SUSPENDED-SEDIMENT SAMPLES}

A U.S. P-46 sampler was used to obtain cross-section suspendedsediment samples at the beginning and end of each sampling period except April 1961, when samples were obtained on only 1 day. All samples were collected by the equal-transit-rate method using 10 verticals in the cross section. The cross-section samples were collected in duplicate; one was analyzed for concentration and the other for size distribution (table 7 ).

TABLE 7.-Size distribution of suspended sediment in cross-section srmples

\begin{tabular}{|c|c|c|c|c|c|c|c|c|c|c|c|c|c|c|c|}
\hline \multirow[b]{3}{*}{ Date } & \multirow{3}{*}{ 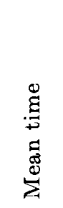 } & \multirow{3}{*}{ 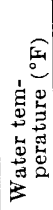 } & \multirow{3}{*}{ 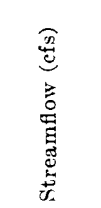 } & \multicolumn{12}{|c|}{ Suspended sediment } \\
\hline & & & & \multirow{2}{*}{ 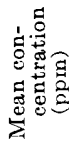 } & \multirow{2}{*}{ 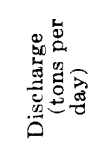 } & \multicolumn{10}{|c|}{ Percent finer than indirated size (mm) } \\
\hline & & & & & & $\begin{array}{l}\mathscr{8} \\
\dot{8} \\
0\end{array}$ & $\begin{array}{l}+1 \\
8 \\
0\end{array}$ & $\begin{array}{l}\infty \\
8 \\
0\end{array}$ & $\begin{array}{l}\stackrel{0}{0} \\
0 \\
0\end{array}$ & $\vec{\varnothing}$ & \begin{tabular}{l}
\multirow{1}{*}{} \\
$\mathscr{8}$ \\
0 \\
0
\end{tabular} & 量 & $\begin{array}{l}0 \\
0 \\
0 \\
0 \\
0 \\
0\end{array}$ & $\begin{array}{l}8 \\
8 \\
0 \\
0\end{array}$ & $\stackrel{8}{8}$ \\
\hline 1961 & & & & & & & & & & & & & & & \\
\hline $\begin{array}{r}\text { Apr. } 21 \ldots \\
1962\end{array}$ & 1040 & 52 & 221,000 & 645 & 385,000 & 36 & 41 & 47 & 57 & 71 & 85 & 90 & 98 & 100 & \\
\hline $\begin{array}{lr}\text { Apr. } 17 & \\
& 25 \\
\text { Oct. } & 8 \\
12 & \ldots\end{array}$ & $\begin{array}{l}1230 \\
1220 \\
1020 \\
1300\end{array}$ & $\begin{array}{l}48 \\
56 \\
65 \\
68\end{array}$ & $\begin{array}{l}363,000 \\
297,000 \\
131,000 \\
136,000\end{array}$ & $\begin{array}{l}455 \\
314 \\
318 \\
328\end{array}$ & $\begin{array}{l}416,000 \\
252,000 \\
112,000 \\
120,000\end{array}$ & $\begin{array}{l}29 \\
33 \\
56 \\
13\end{array}$ & $\begin{array}{l}32 \\
35 \\
56 \\
45\end{array}$ & $\begin{array}{l}39 \\
39 \\
60 \\
53\end{array}$ & $\begin{array}{l}48 \\
48 \\
63 \\
63\end{array}$ & $\begin{array}{l}60 \\
62 \\
82 \\
79\end{array}$ & $\begin{array}{l}69 \\
74 \\
91 \\
91\end{array}$ & $\begin{array}{l}76 \\
84 \\
95 \\
95\end{array}$ & $\begin{array}{r}85 \\
95 \\
100 \\
100\end{array}$ & $\begin{array}{r}99 \\
100 \\
-\cdots\end{array}$ & 100 \\
\hline 1968 & & & & & & & & & & & & & & & \\
\hline Apr. $16 \ldots$ & $\begin{array}{l}1235 \\
0950\end{array}$ & $\begin{array}{l}58 \\
62\end{array}$ & $\begin{array}{l}137,000 \\
124,000\end{array}$ & $\begin{array}{l}318 \\
415\end{array}$ & $\begin{array}{l}118,000 \\
139,000\end{array}$ & $\begin{array}{l}25 \\
19\end{array}$ & $\begin{array}{l}28 \\
20\end{array}$ & -- & $\begin{array}{l}36 \\
31\end{array}$ & 50 & $\begin{array}{l}58 \\
54\end{array}$ & $\begin{array}{l}64 \\
57\end{array}$ & $\begin{array}{r}100 \\
99\end{array}$ & 100 & \\
\hline
\end{tabular}

The concentration of sand is generally very low for the deep flows of the Mississippi River at St. Louis. The measurec concentrations of sand for samples obtained for this study ranged from about 29 parts per million on October 8, 1962, to about 190 ppm on April 20, 1963. The extremes of the variation occurred during the two periods when the streamflows and measured total concentrations were nearly the same. In October 1962, only 9 percent of the suspended sediment was sand, whereas in April 1963 about 44 percent of the suspended sediment was sand; however, the average velocity and average depth (table 1) were about 3.30 feet per second and 25 feet in October 1962 and 3.80 feet per second and 22 feet in April 1963.

\section{BED-MATERIAL SAMPLES}

A BM-54 sampler was used to collect bed-material samples 1 or 2 days before and again 1-4 days after the suspended-sediment data were obtained. Samples of bed material were genorally 
collected at about 30 equally spaced points in the cross section and were analyzed to determine particle-size distribution (table 8).

The size distribution of bed material varied somewhat; generally about 50-60 percent of the sediment was in the size range of $0.062-$ $0.500 \mathrm{~mm}$, and the median size was about $0.42 \mathrm{~mm}$ for the samples obtained in 1961 and in 1962. In April 1963, however, the amount of finer sediment had increased; about 95 percent of the sediment was in the size range of $0.062-0.500 \mathrm{~mm}$, and the median size was about $0.18 \mathrm{~mm}$. The increase of fine sediment ir the bed is reflected in the increase in percentage of sand in suspension. The size distribution of bed material at individual verticals in the cross section was quite variable; however, in 1961 and 1962, the size of bed material in the right half of the channel was larger than that in the left half. In April 1963, when the mean size of bed material in the cross section was only about $0.18 \mathrm{~mm}$, the mean sizes in the right and left half of the channel also were about $0.18 \mathrm{~mm}$.

TABLE 8.-Size distribution of bed material

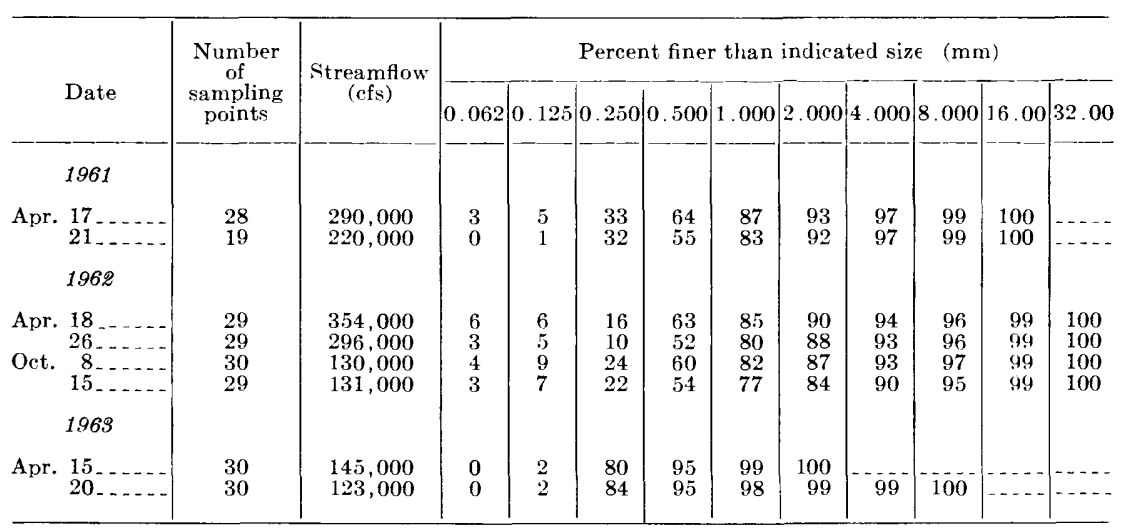

\section{SUMMARY}

Four sets of comprehensive hydraulic and sediment data for deep flows are presented with explanations of field and computational procedures. These data cover a range of mean velocity from 3.3 to 5.6 feet per second, mean depth from 22 to 37 feet, and suspended-sediment concentration from 314 to $928 \mathrm{ppm}$, of which 9-46 percent was sand. The median size of bed material was about $0.42 \mathrm{~mm}$ for three of the sets of measurements but only about $0.18 \mathrm{~mm}$ for the other set. 
Hydraulic data included streamflow measurements, water-surface slopes, cross-sectional areas, and point velocities. E'ergy gradients were computed by adding a velocity head to the rvatersurface elevations. Water-surface slopes and computed energy gradients generally break in the vicinity of mile 179. Longitudinal profiles did not show any bed-configuration changes that might be related to breaks in slope, and the breaks in slope seem likely to have been related to changes in channel width and cross-sectional area in the study reach. The maximum difference between the computed energy gradient over the entire study reach ard the computed energy gradient upstream or downstream from the break was about 17 percent. The average turbulence, or Karman, constant was computed by three methods, but two of the methods were similar because both were computed from average shear velocities and average velocity differences. Measurements ob+ained on consecutive days indicated that data from either streamflowmeasurement notes or from vertical-velocity profiles were satisfactory for computation of the turbulence constant. The turbulence constants computed from streamflow-measurement data ranged from 0.31 to 0.40 and averaged 0.34 ; the constants computed from vertical-velocity profiles ranged from 0.32 to 0.40 and averaged 0.35 .

The sets of sediment data consisted of point samples, crosssection samples, bed-material samples, and water temperatures. Vertical distributions of concentration of various size ranges of sand were used to define $z_{1}$ 's. The $z_{1}$ 's for various size ranges plotted against corresponding fall velocities indicated that the average power of the fall velocity in the relation $z=V_{s} / k u_{*}$ should be about 0.7 . The data also indicated that the relation of $z_{1}$ 's to fall velocity may vary with the mean stream velocity.

\section{LITERATURE CITED}

Anderson, A. G., 1942, Distribution of suspended sediment in a natural stream: Am. Geophys. Union Trans., 23d Ann. Mtg., pt. 2, p. 678-683.

Colby, B. R., and Hembree, C. H., 1955, Computations of total sediment discharge, Niobrara River near Cody, Nebraska: U.S. Geol. Survey WaterSupply Paper 1357, 187 p.

Jordan, P. R., 1965, Fluvial sediment of the Mississippi River at St. Louis, Missouri: U.S. Geol. Survey Water-Supply Paper 1802, 89 p.

Kindsvater, C. E., Carter, R. W., and Tracy, H. J., 1953, Computstion of peak discharge at contractions: U.S. Geol. Survey Circ. 284, 34 p.

U.S. Inter-Agency Committee on Water Resources, 1957, Some fundementals of particle size analysis, Rept. 12 in A study of methods used in measurement and analysis of sediment loads in streams: $55 \mathrm{p}$. 


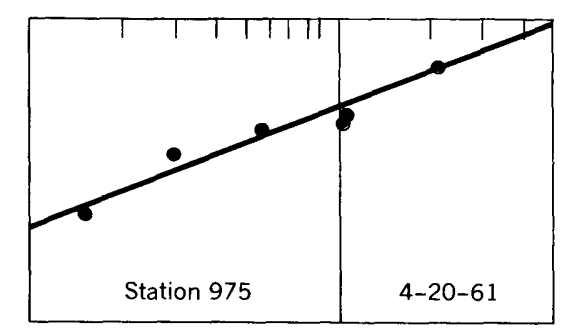

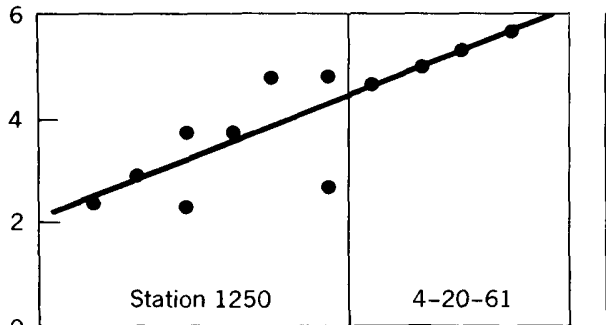
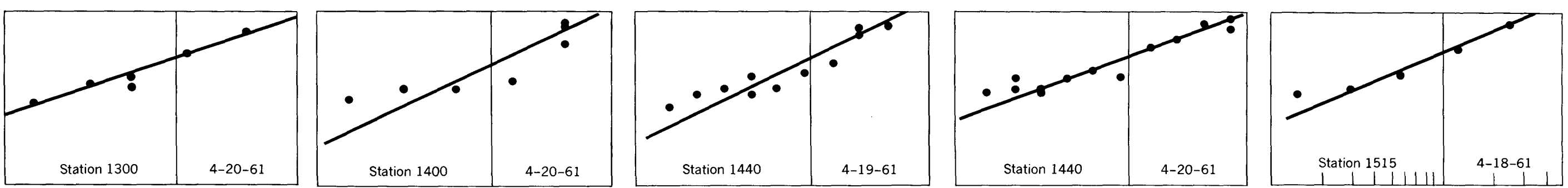

$\sqrt[6]{.69}$
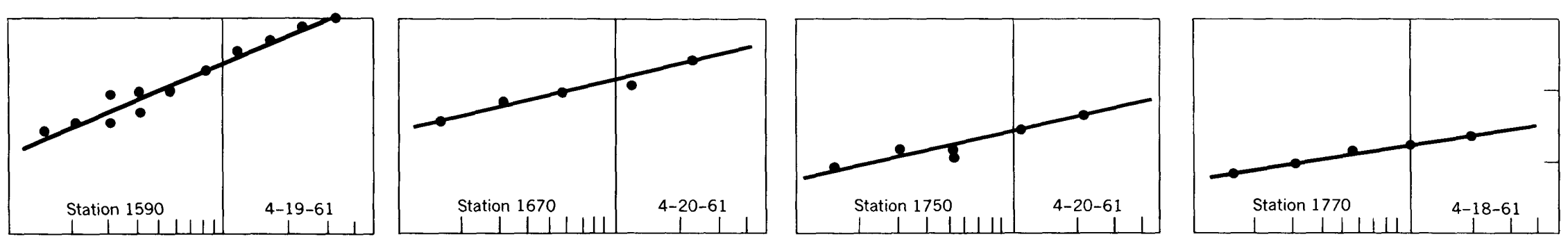

\begin{tabular}{|l|l|l|l|}
\hline & 1 \\
\hline sation 300 & \\
\hline
\end{tabular}
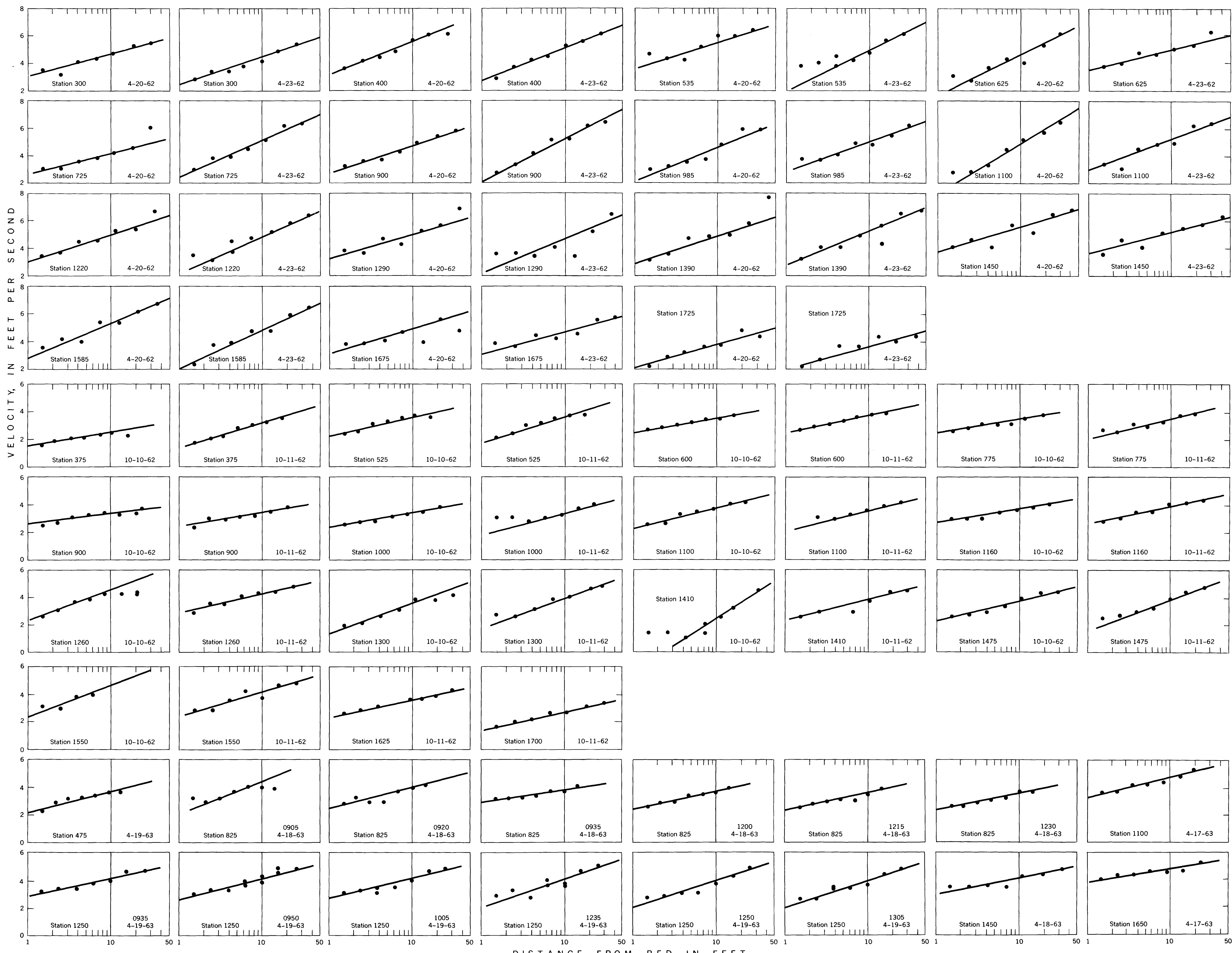

GRAPHS SHOWING VERTICAL DISTRIBUTION OF VELOCITY IN THE MISSISSIPPI RIVER AT ST. LOUIS, MISSOURI 

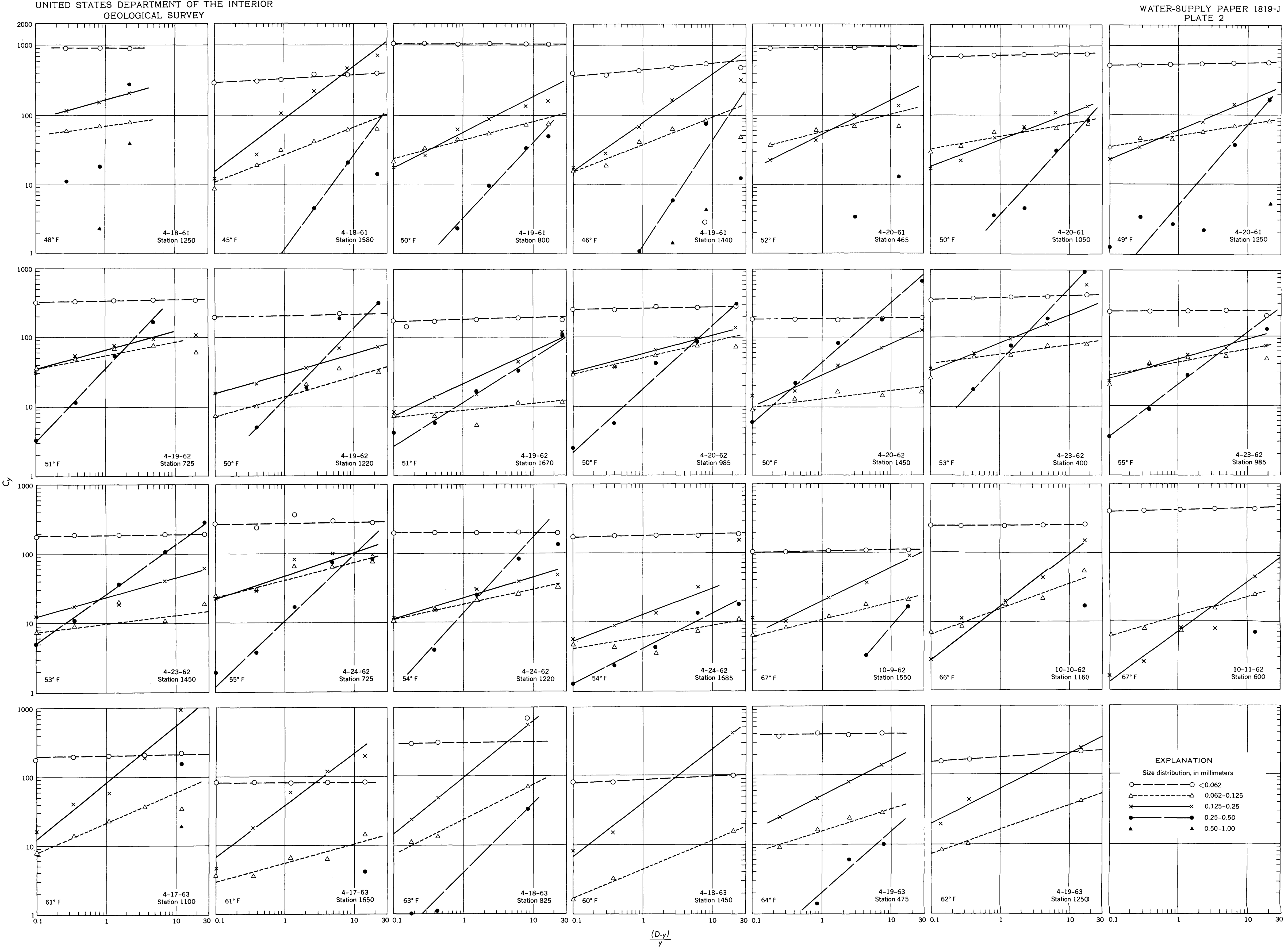

GRAPHS SHOWING VERTICAL DISTRIBUTION OF SUSPENDED SEDIMENT IN THE MISSISSIPPI RIVER AT ST. LOUIS, MISSOURI 

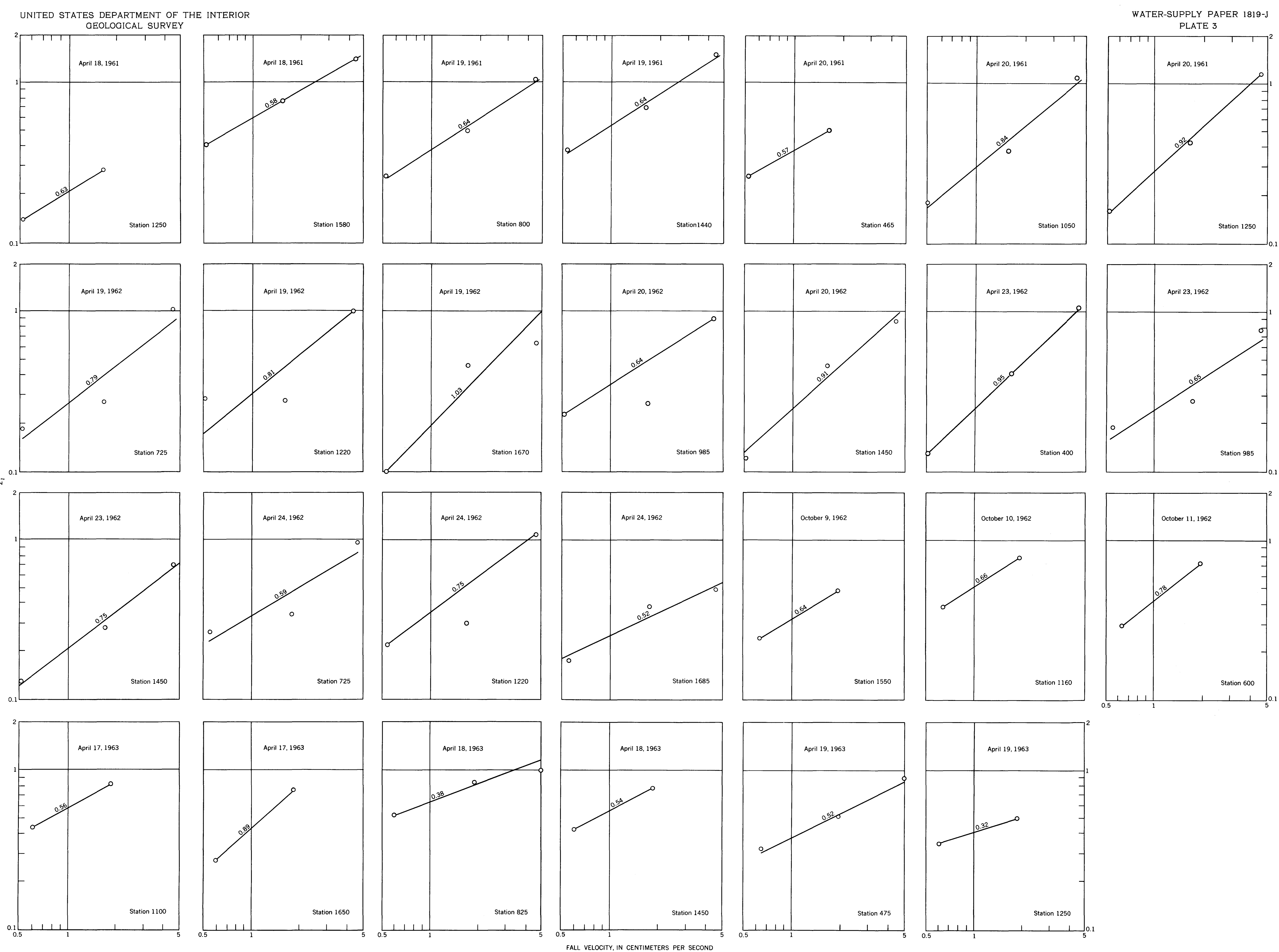

GRAPHS SHOWING RELATION OF $z_{1}$ TO FALL VELOCITY IN THE MISSISSIPPI RIVER AT ST. LOUIS, MISSOURI 